\title{
Maternal obesity drives functional alterations in uterine NK cells
}

\author{
Sofie Perdu, ${ }^{1}$ Barbara Castellana, ${ }^{1,2}$ Yoona Kim, ${ }^{1}$ Kathy Chan, ${ }^{1}$ Lauren DeLuca, ${ }^{1,3}$ \\ and Alexander G. Beristain ${ }^{1,2,3}$ \\ ${ }^{1}$ The Child and Family Research Institute, Vancouver, Canada. ${ }^{2}$ Department of Obstetrics and Gynecology and the \\ ${ }^{3}$ Experimental Medicine Graduate Program, The University of British Columbia, Vancouver, Canada.
}

\begin{abstract}
Over one-fifth of North American women of childbearing age are obese, putting these women at risk for a variety of detrimental chronic diseases. In addition, obesity increases the risk for developing major complications during pregnancy. The mechanisms by which obesity contributes to pregnancy complications and loss remain unknown. Increasing evidence indicates that obesity results in major changes to adipose tissue immune cell composition and function; whether or not obesity also affects immune function in the uterus has not been explored. Here we investigated the effect of obesity on uterine natural killer (UNK) cells, which are essential for uterine artery remodeling and placental development. Using a cohort of obese or lean women, we found that obesity led to a significant reduction in uNK cell numbers accompanied with impaired uterine artery remodeling. uNK cells isolated from obese women had altered expression of genes and pathways associated with extracellular matrix remodeling and growth factor signaling. Specifically, uNK cells were hyper-responsive to PDGF, resulting in overexpression of decorin. Functionally, decorin strongly inhibited placental development by limiting trophoblast survival. Together, these findings establish a potentially new link between obesity and poor pregnancy outcomes, and indicate that obesity-driven changes to uterine-resident immune cells critically impair placental development.
\end{abstract}

Conflict of interest: The authors have declared that no conflict of interest exists.

Submitted: November 18, 2015 Accepted: June 13, 2016 Published: July 21, 2016

Reference information: JCI Insight. 2016;1(11):e85560. doi:10.1172/jici.nsight.85560.

\section{Introduction}

Obesity is a serious and rising cause of obstetrical and perinatal morbidity; in developed countries, over $20 \%$ of women of childbearing age are defined as being obese (BMI $\left.\geq 30 \mathrm{~kg} / \mathrm{m}^{2}\right)(1,2)$. Obese women are about 3 times more likely to develop major complications during pregnancy, such as gestational diabetes or preeclampsia, and excess adiposity increases the risk of preterm birth, which is responsible for $\sim 75 \%$ of the 4 million neonatal deaths annually worldwide $(3,4)$; these disorders likely result from placental dysfunction (5). Studies using animal models have confirmed a role for obesity in causing placental dysfunction defined in part by altered vascular changes within the fetal-maternal environment $(6,7)$. However, the underlying mechanisms linking obesity to poor pregnancy outcomes are unknown.

The condition of obesity is strongly associated with low-grade chronic inflammation (8). Studies using rodent models show that obesity-related stress alters immune cell polarization in metabolically active organs such as adipose tissue, pancreas, and liver (9-11). For example, recent work has highlighted the importance of immunomodulatory factors (i.e., type-2 cytokines) produced by immune cells for maintaining healthy metabolic homeostasis in adipocytes (12-14). In the condition of obesity, adiposederived stress signals recruit and activate local immune sentinels, such as NK cells and cytotoxic CD ${ }^{+}$ $\mathrm{T}$ cells, which in turn produce proinflammatory factors that polarize adipose tissue resident macrophages towards a classical M1-like phenotype (9). The long-term chronicity of these proinflammatory signals leads to increases of proinflammatory cytokines in the blood that impact immune cell function(s) at distal organ sites; effects on glucose intolerance, insulin resistance, and the development of type 2 diabetes are well-accepted examples of this (15). However, the impact of obesity-related chronic inflammation on maternal uterine immune cell composition and function remains unexplored.

Resident NK cells of the uterus, herein referred to as uterine natural killer (uNK) cells, represent as much as $60 \%-70 \%$ of uterine leukocytes in early pregnancy $(16,17)$. Unlike conventional cytotoxic NK cells, uNK cells play central roles in controlling neoangiogenesis, uterine artery remodeling, placental development, and the immune response against fetal antigen (16-18). Studies in mice highlight the importance 
Table 1. Characteristics of sample cohort

\begin{tabular}{lccccccc}
\hline & Age (Year) & Week of Gestation & BMI $\left(\mathbf{k g} / \mathbf{m}^{2}\right)$ & CRP median $(\mu \mathrm{g} / \mathbf{m l})$ & CRP IQR & $\begin{array}{c}\text { Leptin median } \\
(\mathbf{n g} / \mathbf{m l})\end{array}$ & $\begin{array}{c}\text { Leptin IQR } \\
\text { Control }(n=45)\end{array}$ \\
\hline Obese $(n=45)$ & 25 & 8.6 & 21.7 & 0.9 & 0.6 to 2.0 & 10,363 \\
7,013 to 15,652 \\
\hline
\end{tabular}

Abbreviations: CRP, C-reactive protein; IQR, interquartile range.

of uNK cells in pregnancy, where genetic ablation $(19,20)$ or functional inactivation $(21)$ of uNK cell subsets results in profound defects in decidual bed arterial remodeling and impaired angiogenesis. In particular, uNK cell interactions with fetal-derived trophoblasts are important for appropriate uNK cell activation $(22,23)$, resulting in the subsequent secretion of proinflammatory cytokines (IFN- $\gamma$, TNF- $\alpha$, and angiokines [placental growth factor and VEGF-A and -C]) $(21,24)$. These secreted factors help direct uterine blood vessel transformation from narrow-bore vessels under strict vasomotor control to high-capacity/lowpressure conduits lacking encapsulating smooth muscle. Inappropriate or insufficient uNK cell activation is associated with impaired blood vessel transformation and reduced nutrient and gaseous delivery to the placenta and developing fetus (16). Not surprisingly, these physiological inadequacies are strongly associated with life-threatening disorders of pregnancy, including recurrent miscarriage (25), preeclampsia (26), and fetal growth restriction (27). Thus, the importance of uNK cells in establishing a healthy maternal-fetal interface cannot be overstated.

Here we examine the effect of maternal obesity on uNK cell biology. We show that maternal obesity leads to reductions in uNK cell numbers within uterine mucosa, and these changes correlate with impaired vascular remodeling. Using a global gene expression platform, we show that uNK cell expression signatures from obese women are distinct from those of lean women. Namely, uNK cell gene signatures from obese women show enrichment of genes linked with aberrant extracellular matrix (ECM) production and growth factor signaling resembling fibrogenesis. Functional studies highlight PDGFR signaling as a key pathway dysregulated in UNK cells in obese pregnancies, where its activity drives the expression of fibrillar COL3A1 (collagen, type-3, alpha 1) and DCN (decorin). Lastly, we establish that decorin, identified as a gene highly enriched in uNK cells from obese pregnancies, strongly impacts trophoblast health, resulting in placental impairment.

\section{Results}

Maternal obesity is tightly linked with systemic inflammation and uNK cell impairment. To examine the effects of maternal obesity on uNK cell biology, a cohort of women 19-35 years of age undergoing elective pregnancy terminations between 6 and 13 weeks of gestation was established and characterized. From all consenting women, blood, uterine (decidual mucosa), and placental tissues were collected and processed for biochemical and cellular assays. Women were clinically characterized (weight and height, and self-reported variables including exposure to cigarette smoke, diabetes, prescribed antiinflammatory or antihypertensive medications) and categorized into two groups: obese (BMI $\left.\geq 30 \mathrm{~kg} / \mathrm{m}^{2}, n=45\right)$ or control (BMI 20-24.9 $\mathrm{kg} / \mathrm{m}^{2}, n=45$ ) subjects (Table 1 and Supplemental Table 1; supplemental material available online with this article; doi:10.1172/jci.insight.85560DS1). As obesity is associated with low-grade systemic inflammation, we set out to confirm that this connection also holds true for obese women in early pregnancy. ELISA measurements of serum C-reactive protein (CRP; an acute-phase reactant factor of hepatic origin) and the proinflammatory adipokine leptin (synthesized predominately by adipocytes), showed significant positive relationships with increasing BMI (CRP: $r^{2}=0.46, P<0.0001$; leptin: $r^{2}=0.57, P<0.0001$ ) (Table 1 and Figure 1, A and B). Using a threshold for CRP that differentiates chronic low-grade inflammation from acute inflammation and predicts patients' risk in developing heart disease $(\geq 3.0 \mu \mathrm{g} / \mathrm{ml})(28,29)$, we observed that $75.5 \%$ of obese women (34 of 45) showed CRP levels indicative of systemic low-grade inflammation (Figure 1, A and C). In contrast, only 13.3\% (6 of 45) of control subjects had CRP levels above this threshold (Figure 1, A and C). The linear correlation between serum CRP and leptin was strong $\left(r^{2}=0.35\right)$, indicating that the increase in low-grade inflammation measured in obese pregnant women likely stems from increased adiposity (Figure 1D). 
A

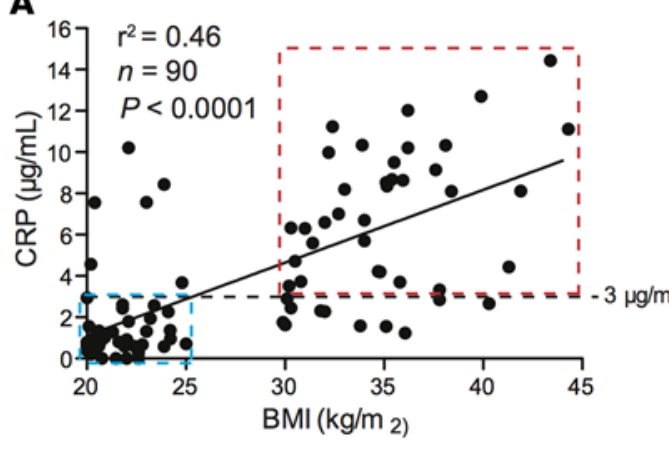

C

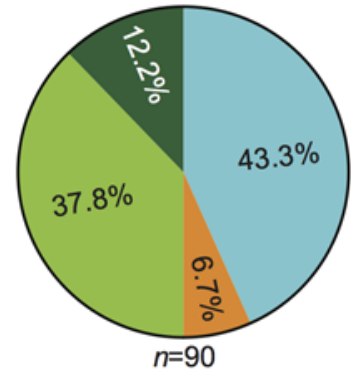

Control: CRP $<3 \mu \mathrm{g} / \mathrm{mL}$ Control: CRP $\geq 3 \mu \mathrm{g} / \mathrm{mL}$ Obese: $C R P \geq 3 \mu \mathrm{g} / \mathrm{mL}$ Obese: CRP $<3 \mu \mathrm{g} / \mathrm{mL}$
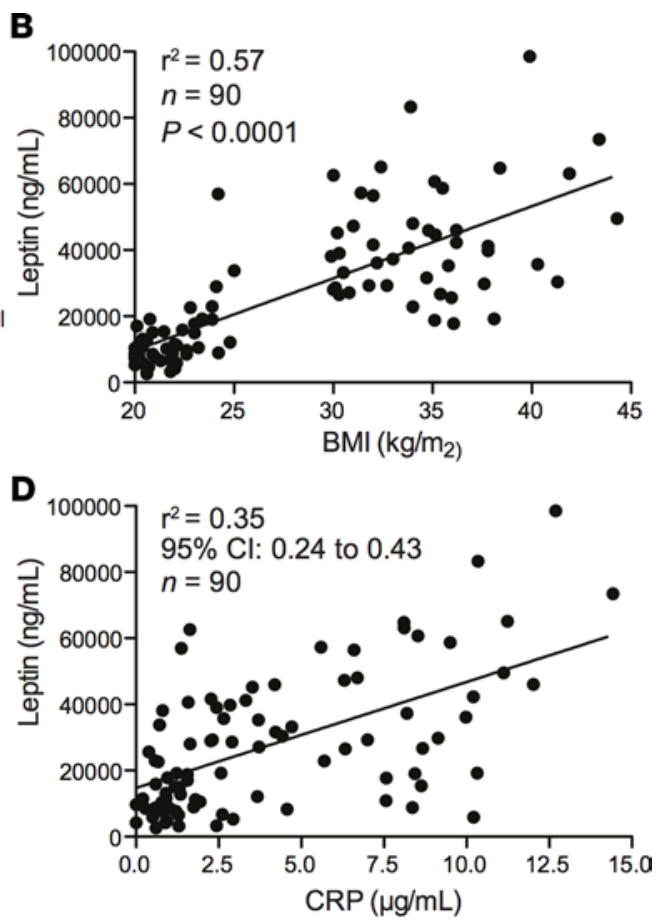

Figure 1. Maternal obesity is associated with low-grade chronic inflammation. (A) Relationship between serum C-reactive protein (CRP) and BMI shown graphically as a line amongst 90 women in early pregnancy. The horizontal line indicates the CRP threshold $(3 \mu \mathrm{g} / \mathrm{ml})$, indicative of low-grade inflammation. The blue hashed box indicates control (lean) BMI samples having CRP values $<3 \mu \mathrm{g} / \mathrm{ml}$; the red hashed box indicates obese BMI samples having CRP values $\geq 3 \mu \mathrm{g} / \mathrm{ml} . r^{2}=$ the coefficient of determination. (B) Relationship between serum leptin and BMI shown graphically as a line. (C) Pie chart depicting proportions of control and obese BMI women presenting with/without elevated serum CRP. (D) Linear regression analysis showing the relationship between serum CRP and leptin between control and obese BMI women. Shown are the $95 \%$ confidence interval $(95 \% \mathrm{CI})$ and the coefficient of determination $\left(r^{2}\right) . P<0.0001$, linear regression statistics.

CD56 ${ }^{\text {bright }} \mathrm{NK}$ cells are the predominant uterine immune cell in pregnancy, playing important roles in orchestrating spiral artery remodeling, angiogenesis, and placental development (16). Because obesity is linked to altered production of immunomodulatory factors known to affect immune cell function (15), we set out to determine if decidual CD56 $6^{\text {bright }} \mathrm{uNK}$ cells are altered in pregnancies of obese women. Using a multicolor flow cytometry strategy, uNK cell proportions from gestationally age-grouped (7-10 weeks) control and obese subjects were measured (Figure 2A). As expected, uNK cells were the predominant immune cell in control decidua, comprising $68.2 \%$ of $\mathrm{CD}_{4} 5^{+}$decidual leukocytes (median $=68.2 \%$, interquartile range $[\mathrm{IQR}]=64.6$ to 76.0 ) (Figure $2 \mathrm{~B})$. In these tissues, a small proportion $(\sim 5 \%)$ of immune cells were CD3-/CD56 $\mathrm{dim} / \mathrm{CD} 16^{+} \mathrm{NK}$ cells, most likely originating from contaminating maternal blood; proportions of CD $56^{\mathrm{dim}} / \mathrm{CD} 16^{+} \mathrm{NK}$ cells did not differ between control and obese women (Supplemental Figure 1A). We did, however, observe a reduction in the proportion of uNK cells in obese subjects compared to controls (obese median $=58.9 \%, \mathrm{IQR}=48.4$ to 70.1 , $P=0.03$ ) (Figure 2, B and C). CD56 median fluorescence intensity (MFI) within the CD56 bright $\mathrm{NK}$ population did not differ between control and obese groups, suggesting that CD56 surface expression is not altered (Supplemental Figure 1, B and C). A possible explanation for the proportional decrease in uNK cells may be related to alterations in cell proliferation. To examine this possibility, we measured proportions of $\mathrm{Ki}^{6} 7^{+} \mathrm{uNK}$ cells in control and obese subjects by flow cytometry and found that proportions of proliferating uNK cells did not differ between BMI groups (Supplemental Figure 1, D and E). In line with our finding showing that high BMI is associated with reduced proportions of $\mathrm{uNK}$ cells, we also observed that CD56 $6^{\text {bright }}$ cell proportions inversely correlate with serum leptin levels $\left(r^{2}=0.33, P=0.0005\right)$ (Supplemental Figure $1 \mathrm{~F}$ ). This relationship held true for CRP; however, the association was modest and not significant $\left(r^{2}=0.11, P=0.06\right)$ (Supplemental Figure 1G). Together, these findings indicate that obesity-linked inflammation in early pregnancy alters uNK cell balance.

To determine if the decreased proportion of uNK cells in obese women is due to absolute reductions in cell numbers, NK cells from decidual tissues at 11-13 weeks of gestation were quantified by immunofluo- 
A

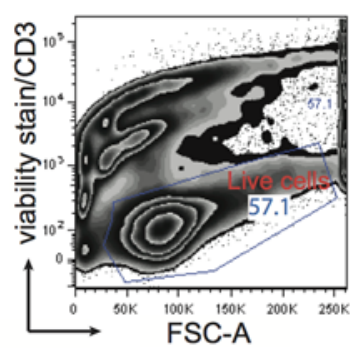

B
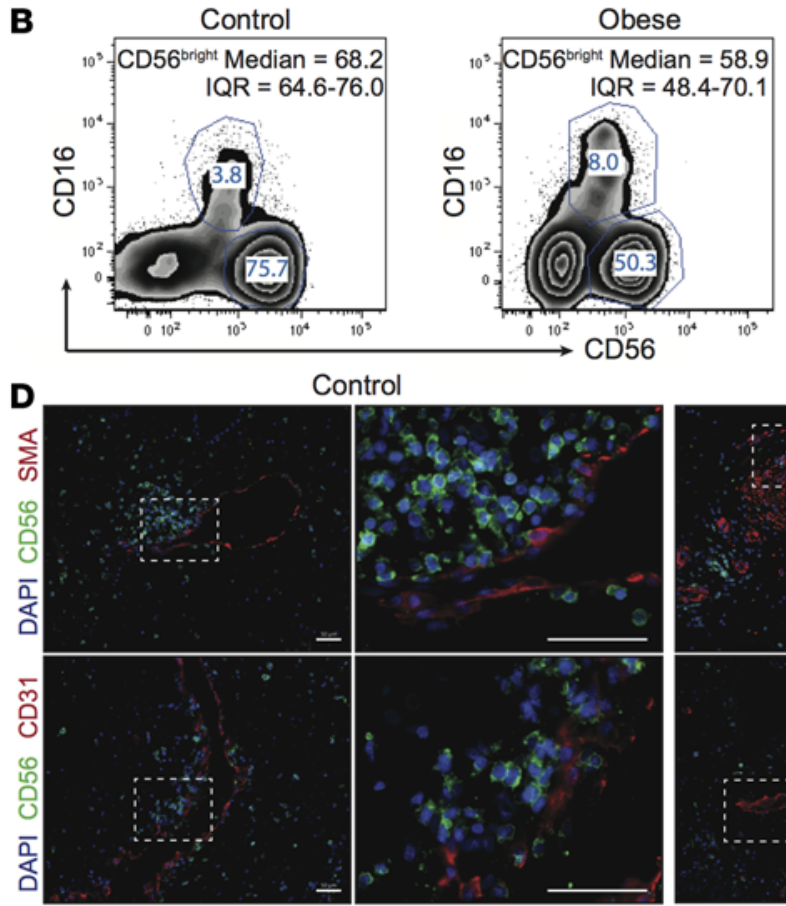

E
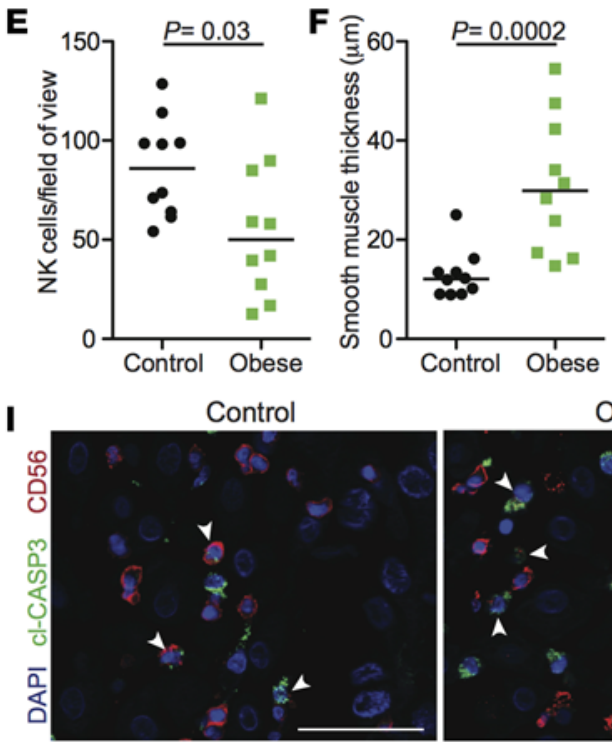
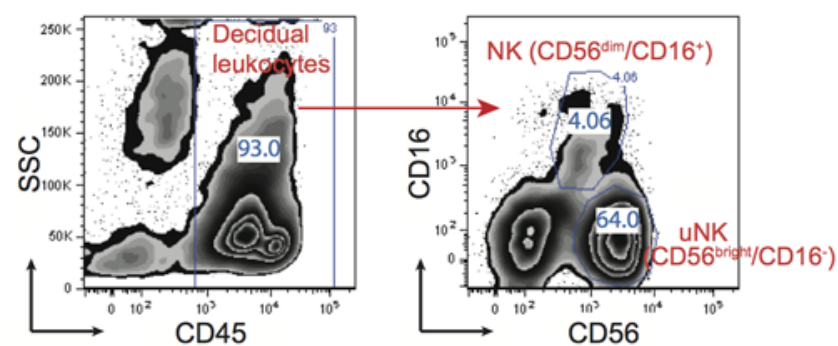

$\rightarrow$

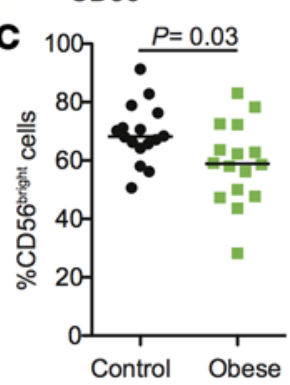

Obese
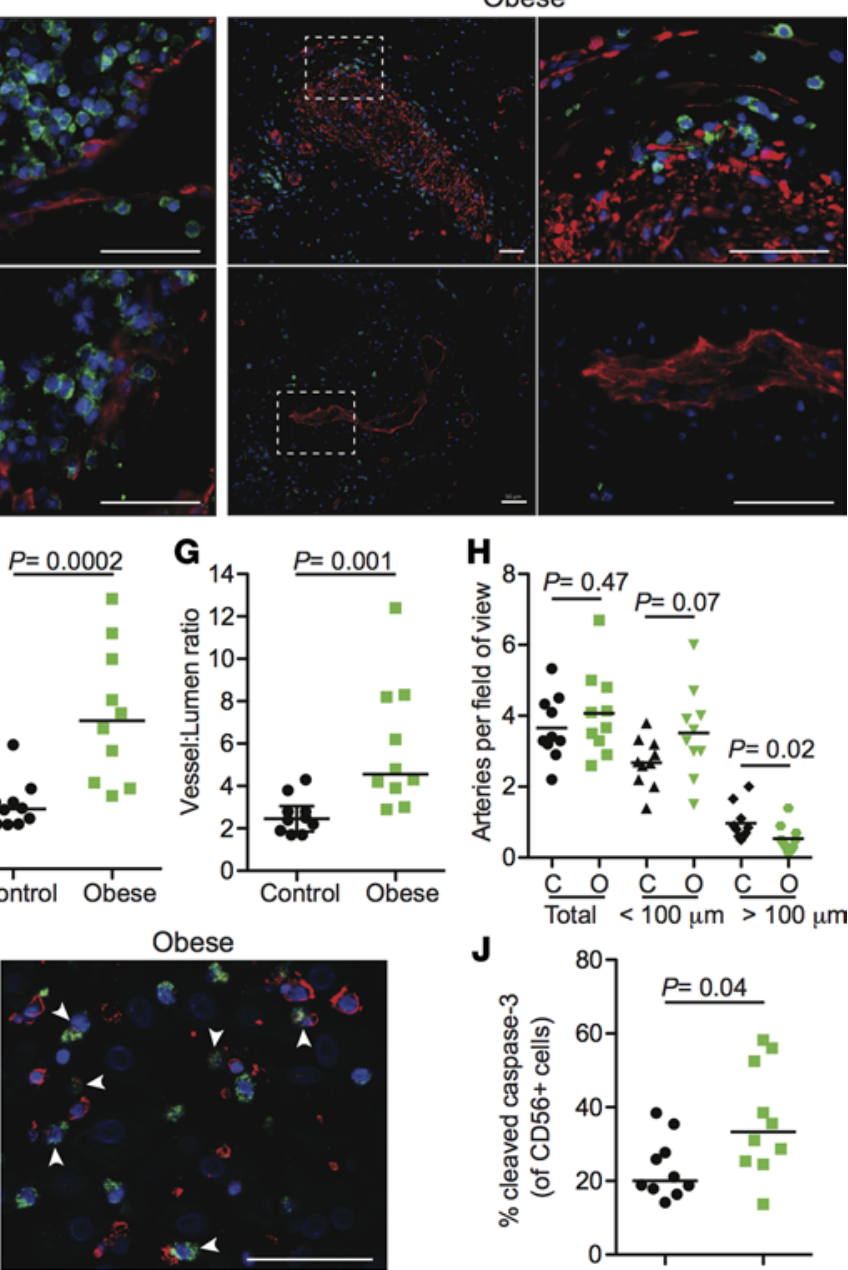

J

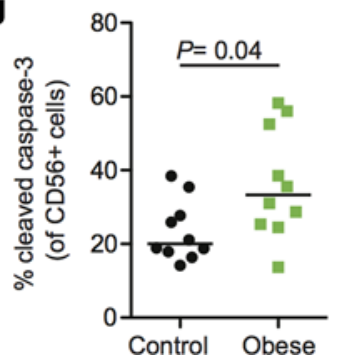

Figure 2. Maternal obesity is linked with reduced numbers of uterine NK (UNK) cells and delayed artery remodeling. (A) Flow cytometry gating strategy to identify and quantify uNK cells in control and obese women. Live decidual leukocytes were selected by excluding nonviable cells and $\mathrm{CD}^{+}$lymphocytes. UNK cell proportions within the $\mathrm{CD} 45^{+}$fraction were quantified by their surface expression of CD56 and CD16 and are presented as proportion of cells. SSC, side scatter. (B) Representative flow cytometry plots of uNK cell proportions in control $(n=16)$ and obese $(n=$ 16) women. Median values of CD56 bright cells are shown within plots. IQR, interquartile range. (C) Scatter plots depicting CD56 bright cell proportions in control and obese women; horizontal line indicates group median. (D) Representative immunofluorescence images of first trimester decidual tissue (11-13 weeks of gestation) from control $(n=10)$ and obese ( $n$ $=10$ ) women immunostained with antibodies directed against CD56 (green), smooth muscle actin (SMA; red), and CD31 (red); DAPI-stained nuclei are labeled blue. Scale bars: $50 \mu \mathrm{m}$. The perforated white box indicates enlarged image. Quantification of (E) absolute uNK cell numbers, (F) arterial smooth muscle thickness, (C) arterial vessel:Iumen ratios and (H) numbers of small $(<100-\mu \mathrm{m}$ diameter) and large $(\geq 100-\mu \mathrm{m}$ diameter) arteries per field of view from decidual specimens of control (C) and obese (O) women. Representative immunofluorescence images (I) and quantification of proportions of apoptosing uNK cells (J) in decidual tissue from control $(n=10)$ and obese $(n=10)$ women. Sections were immunostained with antibodies directed against CD56 (red) and cleaved caspase-3 (cl-CASP3; green); DAPI-stained nuclei are labeled blue. White arrowheads highlight apoptosing uNK cells. Scale bars: $50 \mu \mathrm{m}$. Statistical analyses were performed using a nonparametric 2-tailed MannWhitney $t$ test.

rescence (IF) microscopy. For these analyses, the older gestational age range was selected, as it allows us to quantify uNK cell numbers concurrently with physiological changes in uterine arteries; uterine vascular changes at earlier time points in pregnancy are not as robustly evident. Dual-immunolabeling strategies targeting NK cells (CD56), smooth muscle cells (smooth muscle actin [SMA]), and endothelial cells (CD31) were performed. Consistent with our flow cytometry data, abundant uNK cells were observed in the decidual 
A

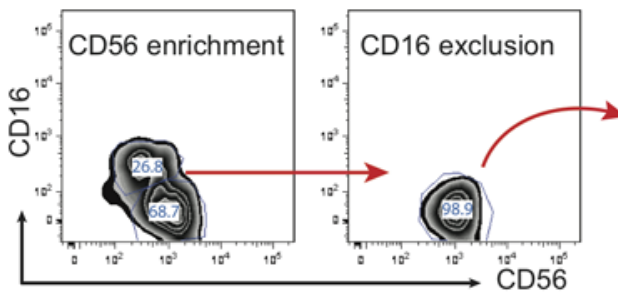

B

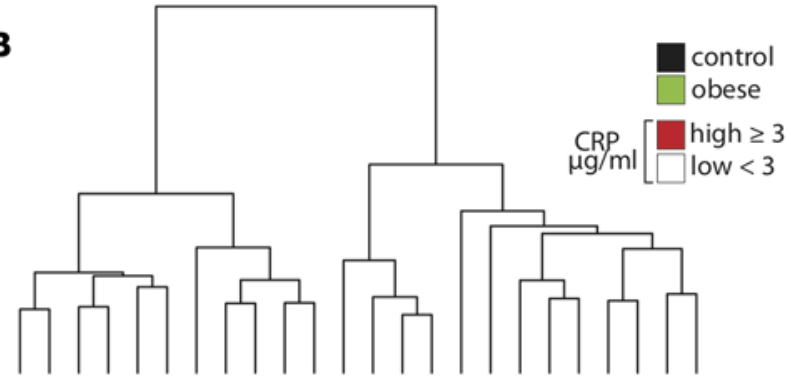

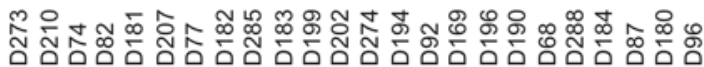
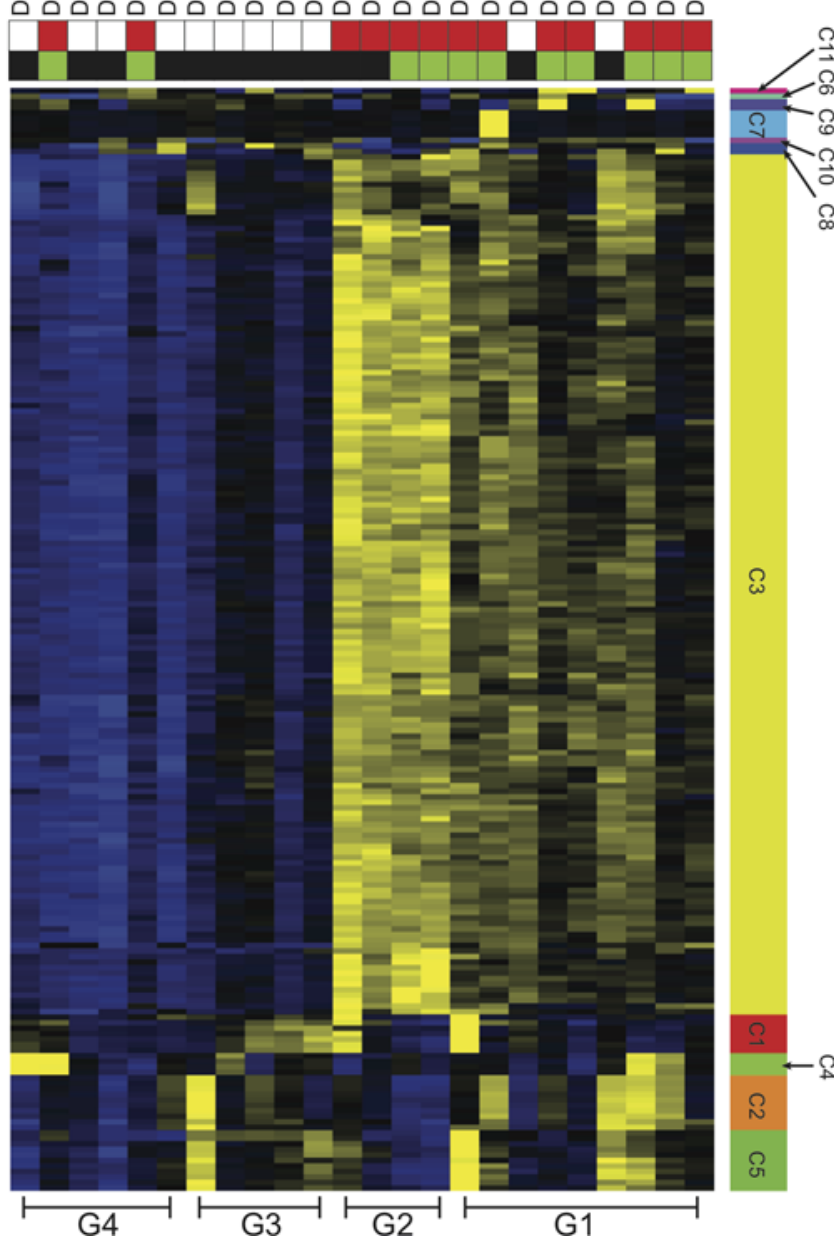

Microarrays Hybridization Data Preprocessing

Computational Analyses
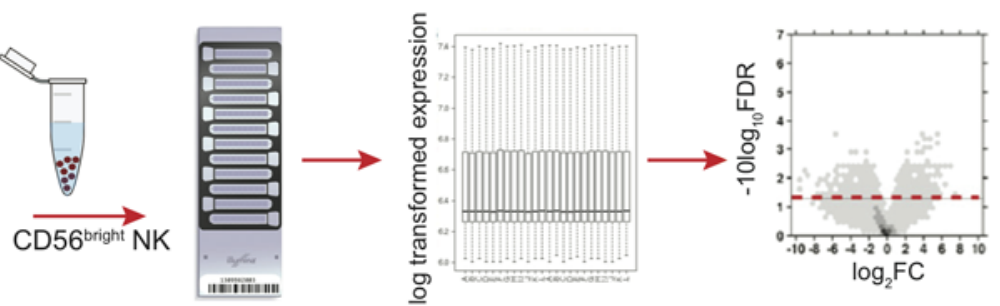

C

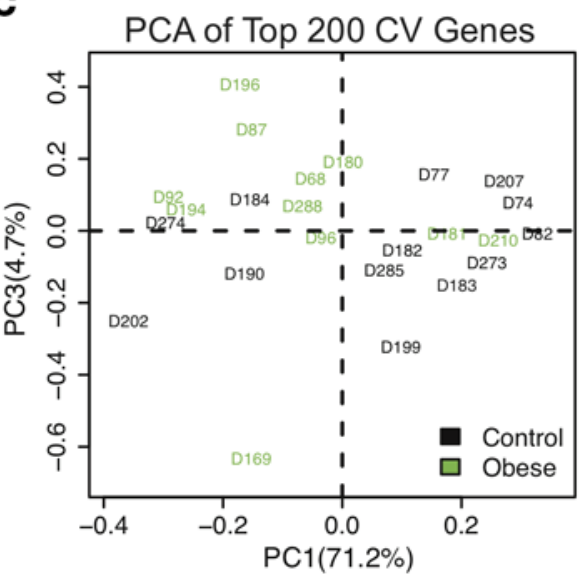

D

Gene Cluster 3

GO: Molecular Function GO: Biological Processes
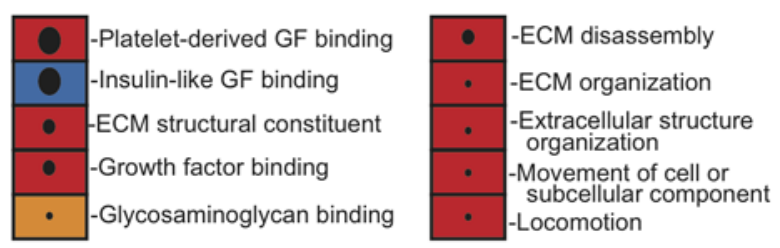

\section{GO: Pathway}

GO: Disease

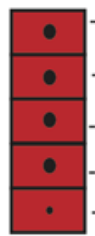

Assembly of collagen fibrils \& other multimeric structure -Collagen biosynthesis \& modifying enzymes -Collagen formation -ECM-receptor interaction -ECM organization

\begin{tabular}{|c|c|}
\hline & -Ulrich congenital \\
muscular dystrophy \\
& -Ehlers-Danlos syndrome \\
type 1 & \\
\hline $\mathbf{O}$ & -Bethlem myopathy \\
\hline$\bullet$ & -Abortion, Spontaneous \\
\hline$\cdot$ & -Endometriosis \\
\hline
\end{tabular}

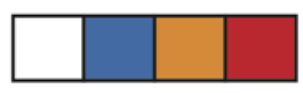

$<10^{-4}<10^{-5}<10^{-6}<10^{-7}$ q value (Bonferroni)

Percent of genes in $\mathrm{GO}$ annotated list

$\geq 50 \%$

- $\geq 10 \% \leq 49 \%$

- $<10 \%$ 
Figure 3. Maternal obesity instructs gene expression-informed uterine NK (UNK) cell clustering. (A) Schema of experimental pipeline starting from NK cell purification, RNA extraction, microarray hybridization, data preprocessing, and computational analyses. (B) Dendrogram depicts hierarchical clustering of control and obese uNK cell samples generated by the 200 high coefficient of variation (high-CV) gene signature where the 24 samples ( $n$ $=13$ control; $n=11$ obese) were separated into 4 groups (C1, G2, G3, and G4). The heatmap displays expression profiles of the top 200 genes with highest CV; the 200 high-CV genes are clustered into 11 gene clusters depicted by different colored bars (C1-C11). Within the heatmap, blue = low expression; black = mid-level expression; yellow = high expression. For each sample, patient BMI and C-reactive protein (CRP) status is indicated above the heatmap (control = black; obese = green; low CRP = white; high CRP = red). (C) Principal component analysis (PCA) of top 200 high-CV genes across 24 uNK cell samples. Control samples are indicated by black type while obese samples are indicated by green. (D) Dotmap depiction of significant pathways enriched in the 154 C3 probe signature determined by ToppGene analyses. FDR values ( $q$ value; Bonferroni) are represented by the color of boxes, and percentage of genes in the GO annotated list are indicated by the size of the circle within each box. Statistical analyses were performed in the R statistical environment using limma library beadarray packages. ECM, extracellular matrix.

mucosa of control subjects (Figure 2D). In these tissues, NK cells preferentially formed large cellular aggregates proximal to remodeled uterine arteries (Figure 2D). Importantly, arterial remodeling in control tissue was extensive, as shown by thin discontinuous SMA/CD31 signals surrounding arterial lumens directly adjacent to large clusters of NK cells (Figure 2D). In contrast with this, uNK cells from obese women were fewer in number (Figure 2, D and E), showed increased thickening of smooth muscle around arteries (Figure 2F), had increased vessel-to-lumen ratios (Figure 2G), and harbored fewer large-bore arteries (all surrogate measurements of reduced vascular remodeling) (Figure $2 \mathrm{H}$ ). Of note, total artery numbers (both small and large) did not differ between BMI groups, suggesting that overall vascularity may not be affected (Figure $2 \mathrm{H})$. To examine if uNK cell survival is altered in obesity, dual IF labeling of CD56 in combination with cleaved caspase-3 antibody highlighted that uNK cell apoptosis is elevated in obese women (Figure 2, I and $\mathrm{J})$. Taken together, these findings indicate that uNK cell-directed uterine arterial remodeling is impaired in obese women in early pregnancy, potentially arising from reduced numbers of uNK cells within decidual tissues; decreased uNK cell numbers may be due to heightened apoptosis.

Maternal obesity generates a unique $u N K$ cell gene expression fingerprint. Our finding that maternal obesity results in reduced numbers of uNK cells and under-remodeled uterine arteries suggests a possible defect in NK cell differentiation and/or activation. To examine the full spectrum of possible gene pathway alterations, microarray gene expression profiles were generated from negatively selected immunomagnetic beadpurified uNK cells from control and obese women (Supplemental Table 2). Decidual leukocyte enrichment via density-gradient centrifugation directly followed by NK cell negative selection and CD16 positive exclusion routinely yielded CD56 $6^{\text {bight }}$ uNK cell purities near 95\% (Figure 3A, Supplemental Figure 2, A and B, and Supplemental Table 2). Unsupervised hierarchical clustering and principal component analysis (PCA) of adjusted global gene expression profiles did not segregate the 13 control uNK cell gene signatures from the 11 obese uNK cell signatures, likely reflecting the inter- and intraheterogeneity in our human cohort (Supplemental Figure 2C). This finding prompted us to investigate the expression difference of a subset of genes between uNK cells from control and obese subjects. Hierarchical clustering of the top-ranked 100 or 200 high-variance probes across all samples (ordered by their coefficient of variance [CV]) separated control and obese uNK cell gene signatures with 75\% accuracy (Supplemental Figure 2D). Using the 200 high-CV probe signature, 4 distinct sample clusters were identified (Groups 1 to 4 ) as shown by the cluster dendrogram (Figure 3B). Groups 1 and 2 (G1/2) showed strong enrichment of obese uNK cell samples ( 9 of 13), whereas Groups 3 and 4 (G3/4) showed enrichment for control BMI samples (9 of 11). Notably, strong concordance with serum CRP levels was observed, underscored by the alignment of 2 control samples (D202 and D274) having elevated CRP with G2 obese samples (Figure 3B). The 200 high-CV probe signature aligned 2 outlier obese samples (D181 and D210) with the control G4 group and 2 control samples (D184 and D190) with the G1 group; sample clustering is graphically illustrated by PCA (Figure 3C). Additionally, hierarchical gene clustering of the 200 high-CV probes identified 11 gene groups (Figure 3B and Supplemental Figure 2E). Gene cluster 3 (C3) was the dominant cluster that consisted of 155 probes primarily upregulated in $\mathrm{CD} 56^{\text {bright }} \mathrm{NK}$ cells from obese subjects. Gene ontology (GO) pathway analysis of C3 genes using ToppGene software (30, 31) (5\% FDR threshold) showed enrichment of molecular functions, biological processes and gene pathways centered on ECM organization, collagen biosynthesis/ assembly, and growth factor binding (Figure 3D and Supplemental Table 3). Interestingly, the C3 gene signature also showed enrichment of genes associated with pregnancy/fertility disorders such as spontaneous abortion and endometriosis (Figure 3D); this association is in accordance with the known elevated risk of developing these disorders with increasing BMI (32). 
A

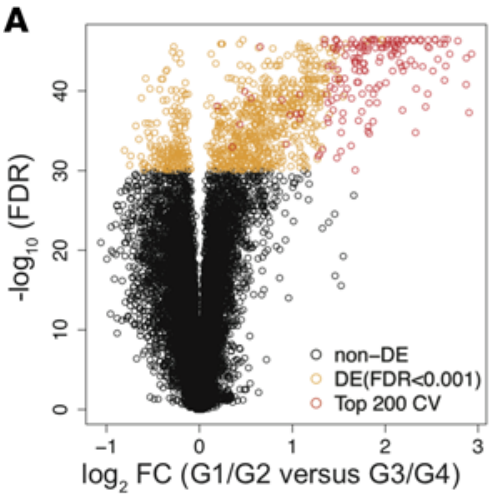

B

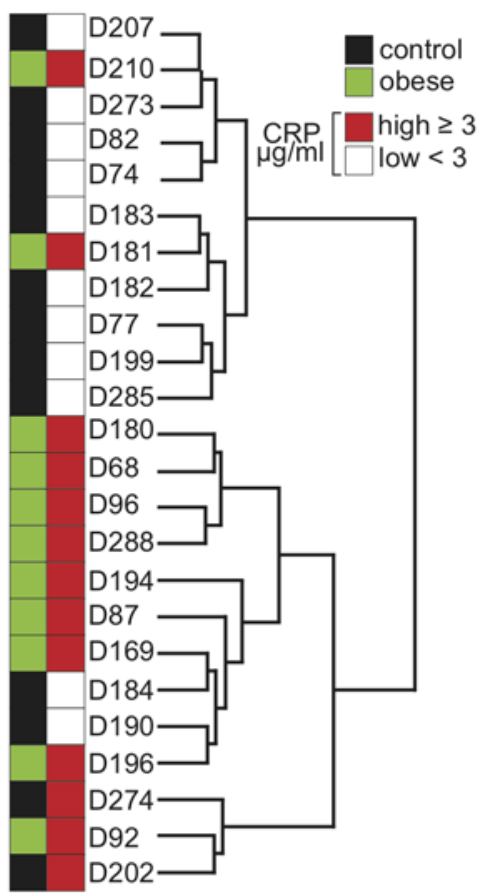

C

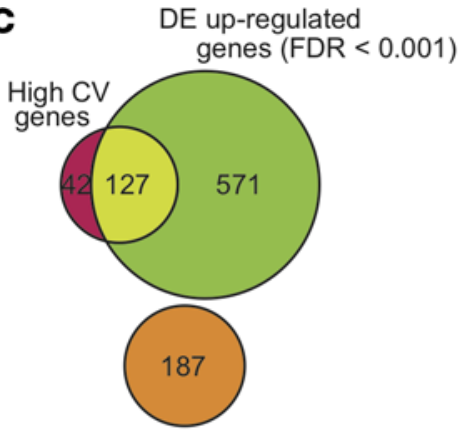

DE down-regulated genes $($ FDR < 0.001)

D

\section{GO: Molecular Function

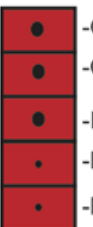 \\ -Growth factor binding \\ -Cell adhesion \\ molecule binding \\ -Integrin binding \\ -Enzyme regulator activity \\ Protein complex binding}

\section{GO: Pathway}
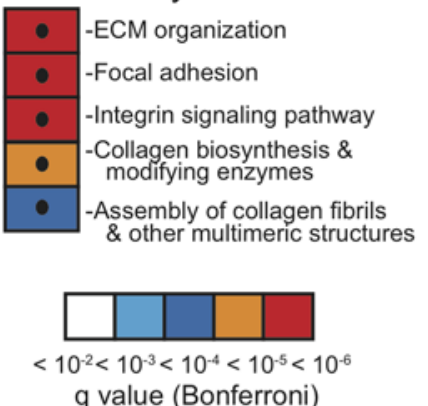

q value (Bonferroni)

\section{GO: Biological Processes}

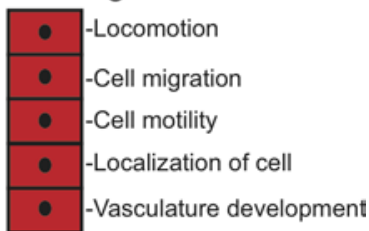

GO: Disease

\begin{tabular}{|l|l}
\hline & -Vascular diseases \\
\hline$\bullet$ & -Endometriosis \\
\hline$\bullet$ & -Prostatic neoplasms \\
\hline$\bullet$ & -Abortion, Spontaneous \\
\hline$\bullet$ & -Neoplasm invasiveness \\
Percent of genes \\
in GO annotated list \\
$\qquad \geq 50 \%$ \\
$\bullet \geq 10 \% \leq 49 \%$ \\
$\bullet<10 \%$
\end{tabular}

E

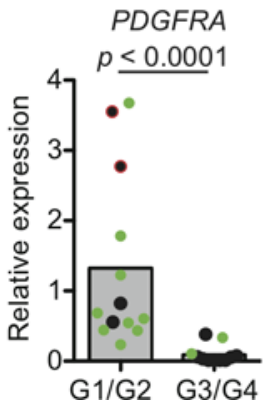

PDGFRB

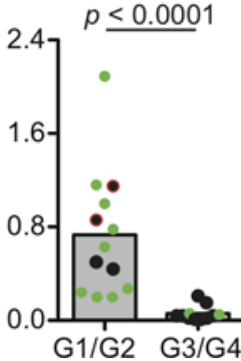

COL1A1

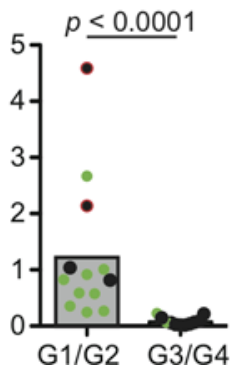

\section{$D C N$}

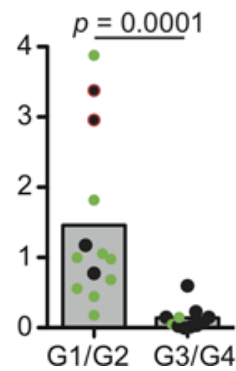

- obese/CRP high

- control/CRP high

- control/CRP low

Figure 4. Differential gene expression (DE) between uterine NK (UNK) cell control- and obese-enriched groups. (A) Volcano plot of differential mRNA levels averaged between $\mathrm{G} 1 / \mathrm{G} 2$ and $\mathrm{G} 3 / \mathrm{G} 4$ sample groups. $x$ axis: coefficients from linear model in log2 scale; $y$ axis: $P$ values adjusted for multiple testing. Black circles indicate probes with an FDR $\geq 0.001$; orange and red circles indicate probes with an FDR $<0.001$; red circles indicate probe overlap with high coefficient of variation (high-CV) probes. (B) Hierarchical clustering of uNK cell samples from obese and control subjects informed by the DE signature (FDR < 0.001) comparing G1/G2 and G3/G4 groups. For each sample, patient BMI and C-reactive protein (CRP) status is indicated above the heatmap (control = black; obese = green; low CRP = white; high CRP = red). (C) Venn diagram showing the number of genes shared between the 200 high-CV list and DE upregulated and downregulated genes (FDR $<0.001$ ). (D) Dotmap depiction of significant pathways enriched in the 885 DE gene signature determined by ToppGene. FDR values ( $q$ value, Bonferroni) are represented by box color, and percentage of genes in the GO annotated list are indicated by circle size. ECM, extracellular matrix. (E) qPCR analysis of PDGFRA, PDGFRB, COL1A1, and DCN in G1/G2 and G3/G4 groups. GAPDH was used for normalization. Individual sample values are indicated by color: black = control/low CRP; red with black = control/high CRP; green = obese/high CRP. Statistical analyses for DE were performed in the R statistical environment; qPCR statistical analyses were performed using a nonparametric 2-tailed Mann-Whitney $t$ test. 
Differential gene expression analysis identifies $u N K$ cell gene pathways altered in obesity. To identify and further define gene networks associated with maternal obesity, we performed a differential gene expression analysis between control-enriched (G3/4) and obese-enriched (G1/2) groups. This analysis identified 1,024 differentially expressed probes $(885$ genes; FDR < 0.001) (Supplemental Table 4). Visualization of these data by volcano plot clearly shows that the majority of differentially expressed genes are overexpressed in the obese-enriched group ( 698 of 885 genes), and that the differentially expressed genes showing the greatest $\log 2$-fold change align strongly with the 200 high-CV gene signature (Figure 4A). Sample hierarchical clustering using the 1,024-probe signature again identified two major groups; one group showed overrepresentation of control uNK cell genes ( 9 of 11), while the other group showed enrichment for gene signatures derived from uNK cells isolated from predominantly obese subjects ( 9 of 13; Figure 4B). Notably, high overlap between high-CV and differentially expressed genes was observed, where 127 high-CV genes overlapped with differentially overexpressed genes (Figure $4 \mathrm{C}$ ). Genes common to both these gene sets included multiple fibrillar collagens (COL1A1, COL1A2, COL3A1, COL4A5, COL5A1, COL5A2, COL6A1, COL6A2, and $C O L 6 A 3)$, insulin-like growth factor binding proteins (IGFBP4, IGFBP5, and IGFBP6), tissue inhibitors of metalloproteinases (TIMP1, TIMP2, and TIMP3), aldehyde dehydrogenases ( $A L D H 1 A 1, A L D H 1 A 2$, and $A L D H 1 A 3$ ), and PDGFRs (PDGFRA and PDGFRB) (Supplemental Table 5). Also common to both the high-CV and differentially expressed gene sets was the gene $D C N$, encoding for the small leucine-rich proteoglycan decorin, which has previously been shown to modulate collagen organization, inhibit trophoblast migration and growth, and is frequently upregulated in proinflammatory fibrotic conditions (33-35).

In addition to BMI as a predictive variable, serum CRP levels were robust in instructing sample clustering. Notably, the clustering of 2 control samples harboring elevated CRP (D274 and D202) with 9 obese samples (all characterized with $\geq 3.0 \mu \mathrm{g} / \mathrm{ml}$ serum CRP) underlined this interaction, whereas only 2 obese uNK cell signatures having elevated CRP clustered with the control G3/G4 group (Figure 4B). Sample segregation informed by serum CRP suggests that systemic inflammation is a key variable in driving altered uNK cell gene expression.

GO analysis of the 885 differentially expressed genes (FDR < 0.001) using ToppGene identified enrichment of molecular pathways and biological processes central to growth factor binding, cell adhesion/integrin biology, cell migration/motility, vascular development, PDGFR signaling, and enzymatic activity (Bonferroni $q$ value $<0.05$; Figure 4D and Supplemental Table 6). Consistent with pathways enriched in the C3 gene cluster, the 885 differentially expressed genes also showed enrichment for biological pathways linked to ECM organization and collagen biosynthesis (Figure 4D and Supplemental Table 6). Real-time PCR (qPCR) analysis of 4 selected genes (COL1A1, DCN, PDGFRA, and PDGFRB) confirmed their overexpression in obese-enriched uNK samples (Figure 4E). Notably, 1 obese uNK cell sample (D92) and the 2 outlier control uNK cell samples harboring high CRP (D274 and D202) consistently showed marked increases in mRNA levels of the 4 genes tested, while expression levels of these genes in samples from the control G3/ G4 group was consistently low (Figure 4E). The ToppGene GO pathway findings, supported in part by the overexpression of specific matrix-associated genes known to be elevated in fibrotic and inflammatory conditions (i.e., DCN, COL1A1, and ACTA2) (36), suggest that an altered in utero environment, driven in part by dysregulated decidual NK cell function/activation, is common in pregnancies of obese women.

PDGFR hyperactivity induces survival signals, but does not affect $u N K$ cell activation. The above gene expression and GO pathway analyses identified PDGFR signaling as a key pathway altered in uNK cells from obese pregnant women. Since the significance of PDGFR signaling in NK biology is poorly defined, we next set out to confirm whether uNK cells from obese women express elevated PDGFR protein levels and whether heightened PDGFR activity affects uNK cellular functions. IF microscopy and flow cytometric analysis confirmed PDGFR localization to NK cells in uterine tissues of both control and obese subjects (Figure 5A and Supplemental Figure 3, A and B). Notably, uNK cell PDGFR- $\alpha$ IF staining in tissue sections of obese samples was more frequently observed and showed greater staining intensity (Figure 5A). While proportions of CD56 $6^{\text {bright }} \mathrm{uNK}$ cells expressing PDGFR- $\beta$ did not differ between control $(n=6)$ and obese $(n=6)$ samples (Supplemental Figure 3B), PDGFR- $\beta$ MFI was modestly higher in the obese group (Figure 5B and Supplemental Figure 3C); this finding is consistent with PDGFR's overexpression at the mRNA level in the majority of uNK cells isolated from obese women. To confirm that PDGFR is functional in uNK cells, we stimulated immunomagnetic bead-purified uNK cells from control $(n=3)$ and obese $(n=3)$ women with PDGF-BB (a PDGFR ligand capable of activating both $\alpha$ - and $\beta$-type PDGFRs), and examined activation of canonical PDGFR pathways via immunoblotting. PDGF-BB treatment resulted in 


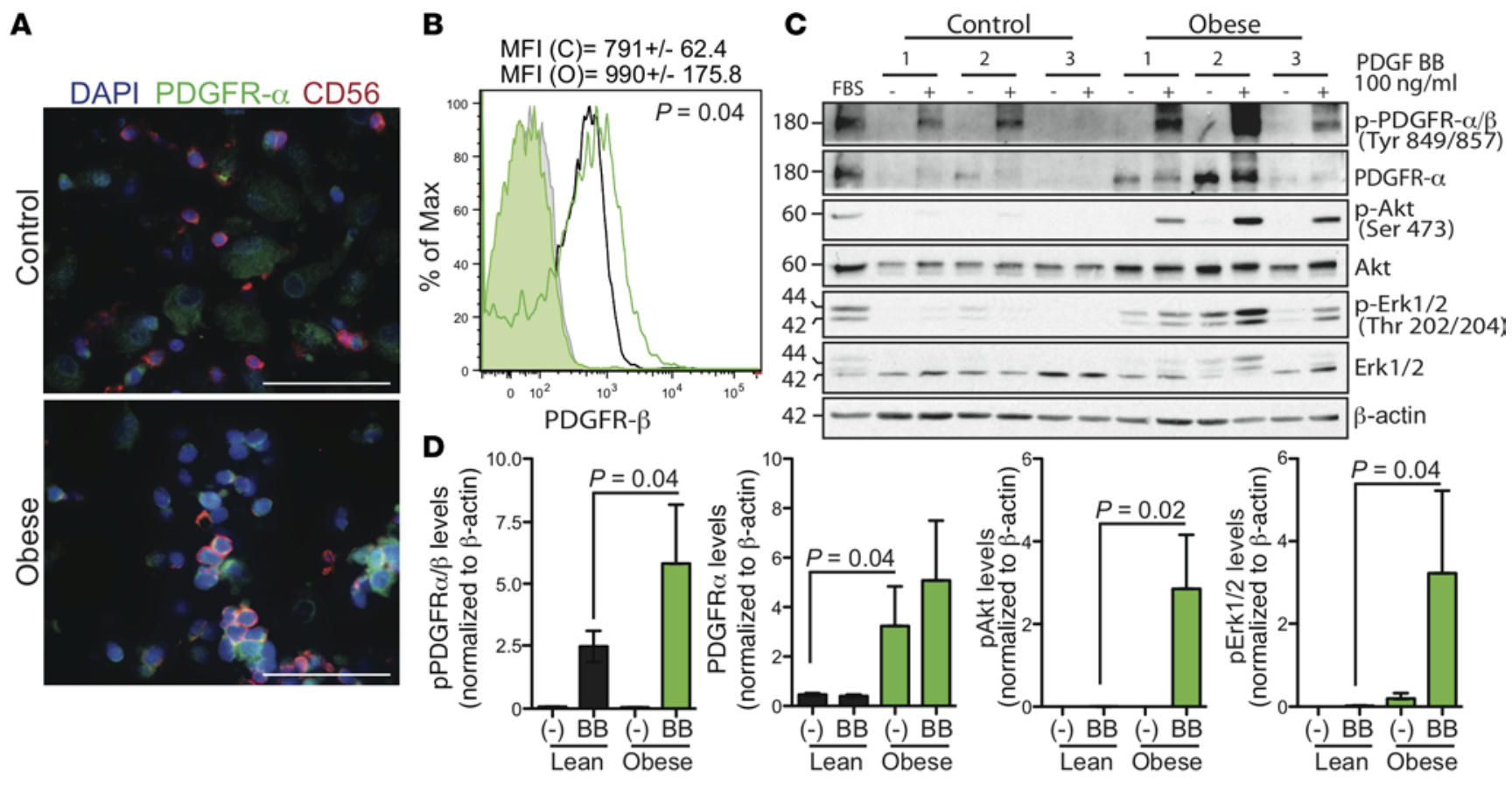

E
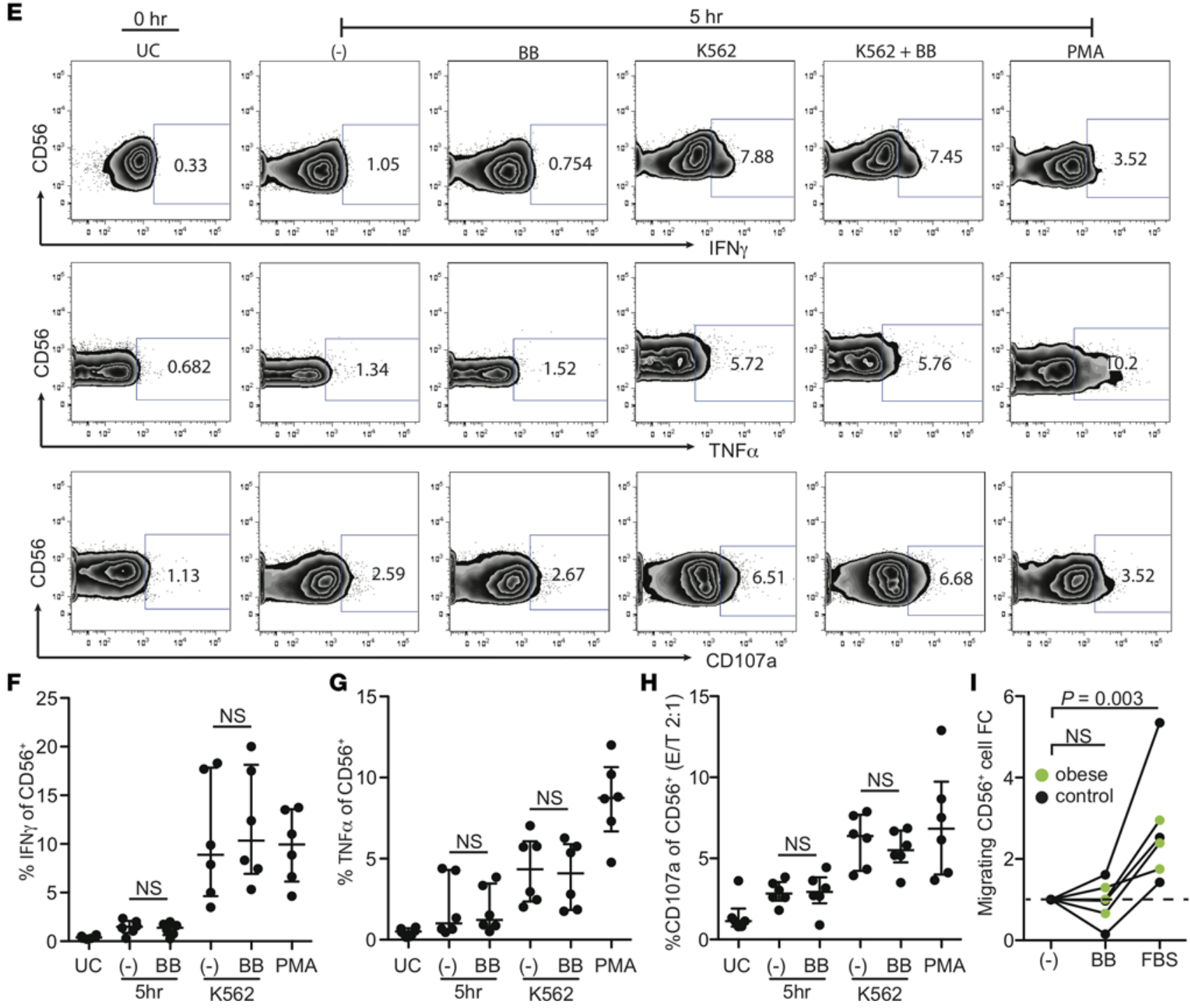
Figure 5. PDGFR induces survival signals in uterine NK (UNK) cells but does not alter cytokine expression, cytotoxicity, or migration. (A) Representative images of decidual tissues at 11-13 weeks of gestation from control $(n=10)$ and obese $(n=10)$ women immunostained for CD56 (red) and PDCFR- $\alpha$ (green). Nuclei are labeled with DAPI (blue). Scale bars: $50 \mu \mathrm{m}$. (B) Representative flow cytometry histogram showing PDCFR- $\beta$ cell-surface intensity on uNK cells from control or obese women. The median fluorescence intensity (MFI) \pm SEM between control $(n=6)$ and obese $(n=6)$ uNK cells is shown above plot: solid color indicates the fluorescence minus one baseline signal. Immunoblots (C) and densitometric quantification (D) depicting activation of PDGFR- $\alpha$, Erk1/2, and Akt after 10 minutes of stimulation with $100 \mathrm{ng} / \mathrm{ml} \mathrm{PDGF-BB} \mathrm{(BB;} \mathrm{a} \mathrm{PDGFR} \mathrm{ligand} \mathrm{capable} \mathrm{of} \mathrm{activating}$ both $\alpha$ - and $\beta$-type PDGFRs) in control $(n=3)$ and obese $(n=3)$ uNK cells. Molecular weights $(\mathrm{kDa})$ are shown to the left and $\beta$-actin indicates loading control. (E) Representative flow cytometry plots of intracellular IFN- $\gamma$ and TNF- $\alpha$, as well as surface expression of CD107a in $1 \times 10^{6}$ CD56 $6^{+}$uNK cells either untreated (-) or treated with $30 \mathrm{ng} / \mathrm{ml} \mathrm{PDGF-BB} \mathrm{for} 5$ hours in the presence or absence of K562 target cells (effector/target ratio 2:1). UC = untreated control. Proportion (\%) of $\mathrm{CD} 56^{+} \mathrm{uNK}$ cells showing positivity for these makers is indicated within plots. Scatter plots show proportional

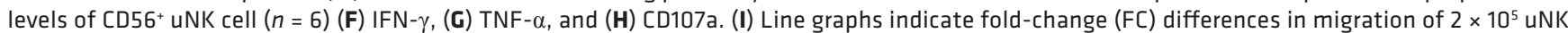
cells treated or untreated with PDGF-BB over 3 hours. UNK cells were purified from control (black, $n=3$ ) or obese (green, $n=3$ ) subjects. FBS treatment served as a positive control. Results are presented as scatter plots with indicated median and interquartile range. Statistical analyses between groups were performed using a nonparametric 2-tailed Mann-Whitney $t$ test. NS, not significant; PMA, phorbol 12-myristate 13-acetate.

activation of PDGFR- $\alpha$ in uNK cells from both control and obese subjects, as shown by phosphorylation of PDGFR- $\alpha$ 's Tyr 849 residue (Figure 5, C and D; full, uncut gels available in Supplemental Figure 4). Notably, both total and phosphorylated PDGFR- $\alpha$ levels were higher in uNK cells from obese women (Figure 5D). Consistent with an increase in PDGFR activity, PDGF-BB stimulation also led to profound induction of phospho-Akt (Ser 473) and phospho-Erk1/2 (Thr 202/204) in the 3 uNK cells examined from obese subjects, while pathway induction was less pronounced in control cells (Figure 5, C and D). Together, these analyses demonstrate that PDGFR signaling is intact and elevated in uNK cells of obese women.

The effect of PDGFR activation on key uNK cell functional readouts was next examined. We specifically measured the ability of PDGF-BB to induce uNK cell cytokine production (TNF- $\alpha$ and IFN- $\gamma$ ), degranulation/cytotoxicity (measured via CD107a surface expression), and migration (Supplemental Figure 3D). Intracellular expression of TNF- $\alpha$ and IFN- $\gamma$ in PDGF-BB-treated uNK cells, as measured by flow cytometry, did not differ from levels in untreated controls (Figure 5, E-G). In these experiments, uNK cells from only obese subjects were used, as levels of PDGFR were expected to be higher than in control subjects and the uNK cells were therefore expected to be more sensitive to PDGF treatment; PMA/ionomycin treatment served as a positive control. Cytokine levels in uNK cells cocultured with K562 target cells were higher than in unstimulated baseline conditions; however, PDGF-BB treatment neither potentiated nor inhibited this induction (Figure 5, E-G). uNK cell degranulation/activation measured by the cell-surface marker CD107a in cells cocultured with K562 target cells was also not affected by PDGF-BB treatment (Figure $5 \mathrm{H}$ ).

In other cell systems (i.e., in cancer and smooth muscle), PDGF promotes cell migration $(37,38)$. Since NK cell infiltration from peripheral blood into the uterus is thought to be a major source of uNK cells in pregnancy, we examined if PDGFR signaling could induce uNK cell motility using a Transwell system. Surprisingly, PDGF-BB, serving as a chemoattractant, had no effect on the migration of uNK cells isolated from either control $(n=3)$ or obese $(n=3)$ women; however, fetal bovine serum serving as a positive control did promote uNK cell migration (Figure 5I). The results of these experiments suggest that PDGFR signaling does not affect typical NK cellular readouts such as proinflammatory cytokine production or cytotoxicity, but may promote cell survival as shown by enhanced Akt and Erk1/2 activity.

PDGFR signaling drives expression of ECM genes in uNK cells. Our microarray-informed finding that uNK cells from obese women are more likely to overexpress COL1A1 as well as other fibrillar collagens and ECMassociated genes was unexpected. Upregulation of ECM and fibrous connective tissue genes is strongly associated with chronic inflammation and fibrotic-like conditions in the liver and lung (36). To investigate the potential contribution of aberrant collagen deposition by uNK cells within the uterine mucosa of obese women (uNK cells comprise $\sim 30 \%$ of all decidual cells in early pregnancy), dual IF microscopy was performed probing for CD56 (NK cell marker) and alpha-1 type I collagen (collagen-I). Collagen-I was broadly localized throughout decidual tissues of both BMI groups, forming network-like structures proximal to and surrounding decidualized stromal cells (Figure 6A). Collagen-I also showed woven-like patterns around unremodeled uterine blood vessels that appeared compact and dense (Figure 6A). Within and proximal to uNK cells, collagen-I colocalization was nearly absent in tissues from controls; however, diffuse expression of collagen-I was frequently observed amongst uNK cells in obese subjects, suggesting that uNK cells in obesity are actively contributing to collagen matrix deposition (Figure 6A).

In tissue fibroblasts and vascular smooth muscle cells, fibrillar collagen overexpression is in part reg- 
A
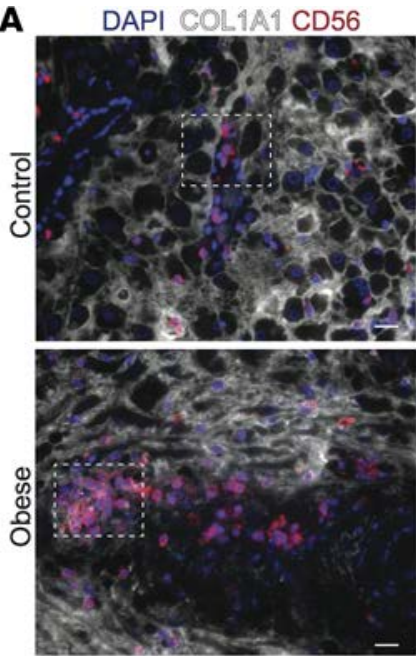

\section{B}
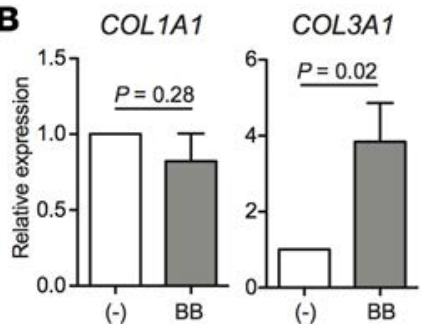
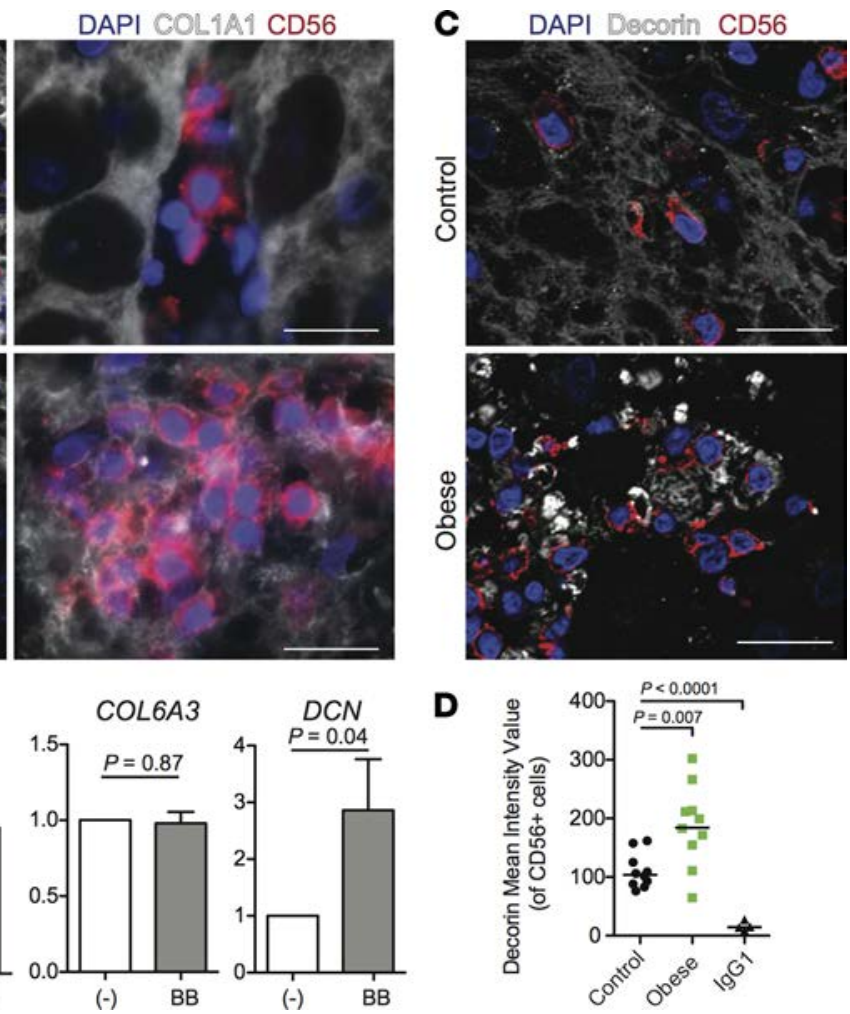

Figure 6. PDGF controls expression of collagen-associated genes in uterine NK (UNK) cells. (A) Representative images of decidual tissues at 11-13 weeks of gestation from control $(n=10)$ and obese $(n=10)$ women immunostained for CD56 (red) and collagen-1 (white). Nuclei are labeled with DAPI (blue). The perforated white box indicates the enlarged image. (B) qPCR analysis of COL1A1, COL3A1, COL6A3, and DCN in $1 \times 10^{6}$ uNK cells treated or untreated (-) with $30 \mathrm{ng} / \mathrm{ml}$ PDGF-BB (BB; a PDGFR ligand capable of activating both $\alpha$ - and $\beta$-type PDGFRs) for 12 hours. Gene expression experiments were performed in triplicate and repeated on uNK cells isolated from 3 distinct decidual leukocyte preparations $(n=3)$. Data presented as the mean \pm SD and statistical analyses were performed using 2-tailed Wilcoxon signed-rank $t$ tests. (C) Representative immunofluorescence images of decidual tissues at 11-13 weeks of gestation from control and obese women immunostained with antibodies directed against CD56 (red) and decorin (white). Scale bars: $25 \mu \mathrm{m}$. (D) Scatter plots show decorin mean channel intensities in uNK cells from control $(n=10)$ and obese $(n=10)$ women; IgG1 staining in 4 obese tissues establishes background signal. Horizontal lines indicate group median. Statistical analyses between groups were performed using a nonparametric 2-tailed Mann-Whitney $t$ test.

ulated by PDGFR signaling (39). Our finding that PDGFRA and PDGFRB gene levels and signaling pathways are intact and hyperactive in obese $\mathrm{UNK}$ cells prompted us to examine if PDGFR regulates expression of collagens and collagen-associated genes. Isolated uNK cells from obese subjects were stimulated with or without $30 \mathrm{ng} / \mathrm{ml}$ PDGF-BB for 12 hours, after which mRNA levels of 4 collagen and collagen-associated ECM genes previously shown to be overexpressed in the obese G1/G2 group (COL1A1, COL3A1, COL6A3, and $D C N$ ) were measured by qPCR analysis. PDGF-BB treatment did not affect COL1A1 or COL6A3 levels, although stimulation did increase the expression of $C O L 3 A 1$ and $D C N$ by approximately 3.0-fold over untreated levels (Figure 6B). IF microscopy within decidual tissues of control samples showed decorin localization proximal to stromal cells, with low signal surrounding uNK cells (Figure 6C). In contrast, abundant decorin signal was seen proximal to uNK cells in obese women (Figure 6C), where the mean uNK-derived decorin signal was higher than in control uNK cells. Together, these findings provide evidence that elevated PDGFR signaling in UNK cells of obese pregnant women may be in part responsible for the increased production (COL3A1) and/or remodeling (via $D C N$ ) of fibrillar collagen within the fetalmaternal interface.

Decorin blocks placental trophoblast outgrowth. DCN was shown to be one of the highest rank-ordered genes overexpressed in the obese G1/G2 uNK cell cohort (Supplemental Table 4). Furthermore, our finding that $D C N$ mRNA levels in uNK cells are controlled via PDGFR establishes a possible mechanism whereby decorin levels are regulated in obesity. uNK cells not only play critical roles in pregnancy by promoting 
A

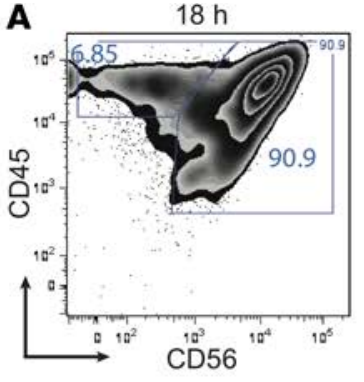

D
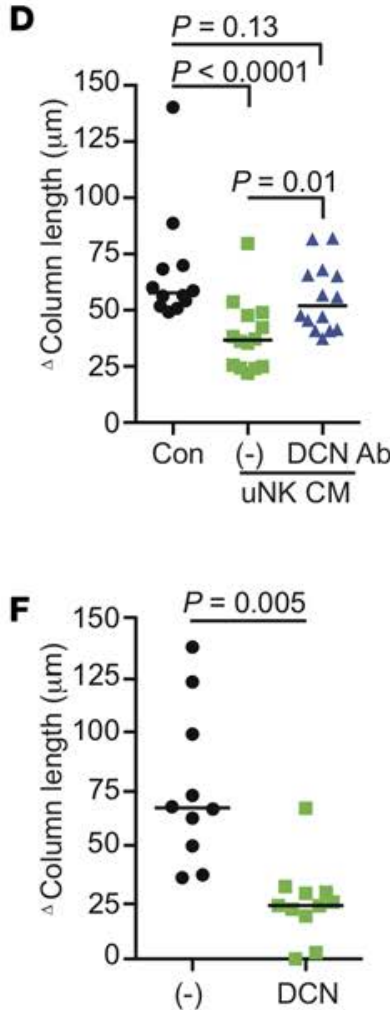

H

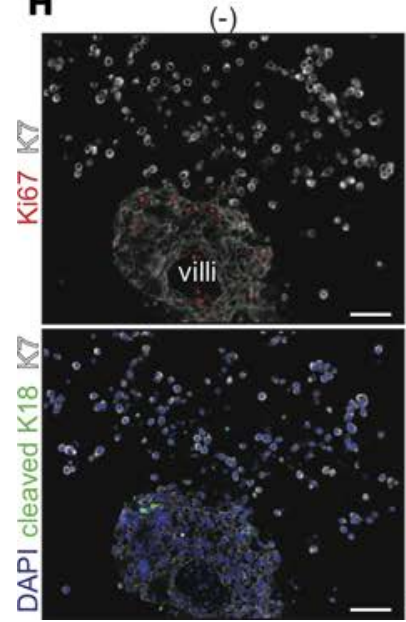

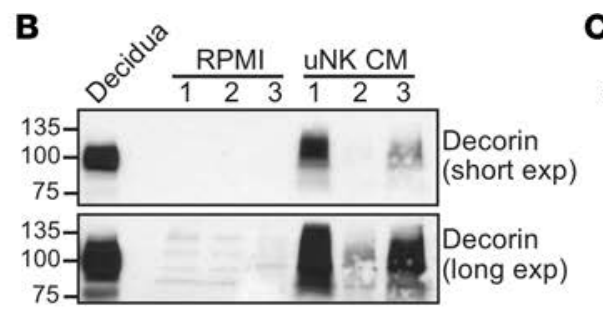

C

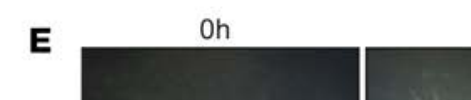

$48 h$
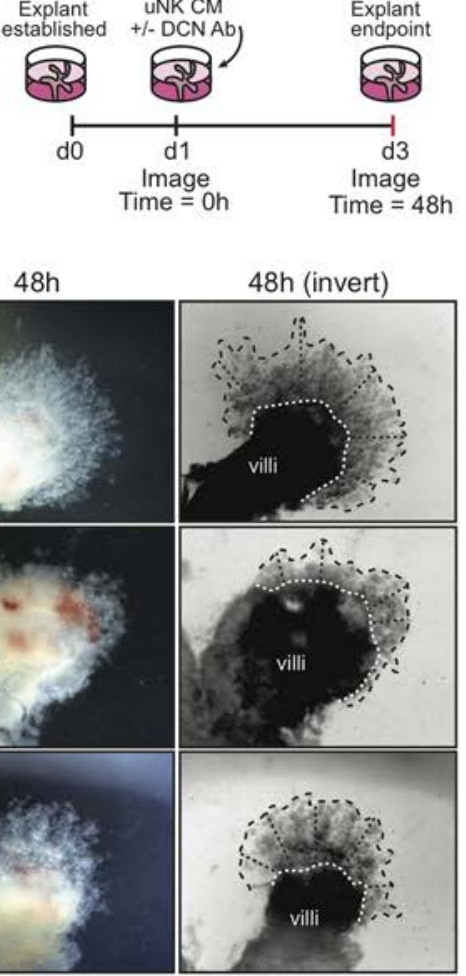

G

Oh

$48 \mathrm{~h}$

48h (invert)
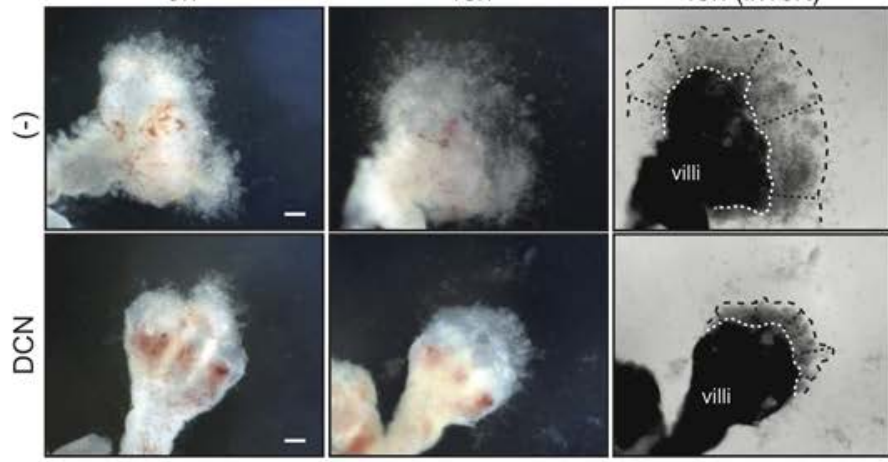

DCN
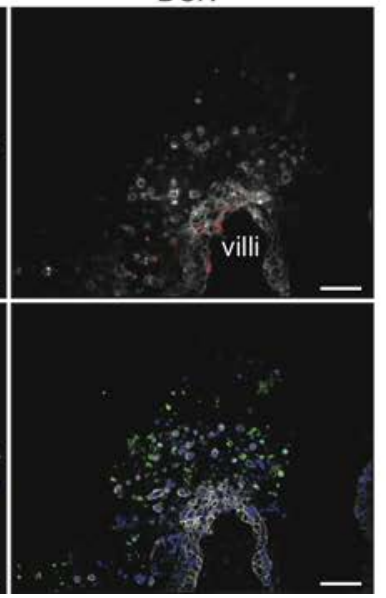

J Cleaved K18

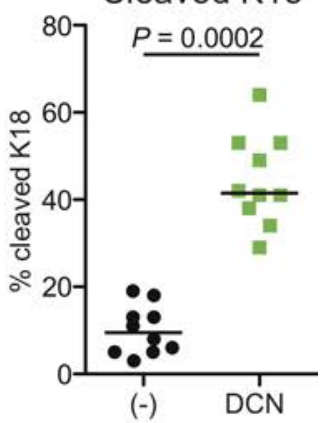


Figure 7. Decorin inhibits placental column outgrowth. (A) Representative flow cytometry plots showing uterine NK (uNK) cell purity following 18 hours of culture. (B) Immunoblot showing decorin in uNK cell conditioned media (CM) from 3 distinct obese uNK cell cultures. Decidual tissue (Decidua) and control media (RPMI) function as positive and negative controls, respectively. Molecular weights ( $\mathrm{kDa}$ ) are shown to the left. (C) A schematic representation of the experimental procedure and timeline: $C M$ = conditioned medium; DCN Ab = decorin-neutralizing antibody; $d$ = day. (D) Quantification of extravillous trophoblast (EVT) column outgrowth in control (Con) and uNK cell CM-treated (UNK CM) placental explants in the presence or absence of a decorin-blocking antibody (DCN Ab). Outgrowth is calculated as the difference in column length $(\mu \mathrm{m})$ at 48 and 0 hours. Explant assays were performed in duplicate using placental villi from 3 independent placentae ( $n=12$ to 14 columns per condition). (E) Representative images of placental villous explants cultured in contro media, or in UNK CM with/without DCN Ab at 0 and 48 hours of culture. Inverted images of explants (invert) provide better contrast, where "villi" indicates placental villi, and hashed lines represent the EVT column base (white) and invasive extremity (black). Scale bars: $100 \mu \mathrm{m}$. Quantification (F) and representative images (C) of EVT column outgrowth following treatment with ( $n=10$ columns) or without ( $n=10$ columns) $20 \mu \mathrm{g} / \mathrm{ml}$ recombinant decorin (DCN). Placental explants were established from 4 independent placentae. (H) Representative images showing proliferative (Ki67+; red) and apoptotic (caspasecleaved cytokeratin-18+ [K18+]; green) trophoblasts within EVT columns. The trophoblast marker keratin-7 (K7) is labeled white and nuclei are stained with DAPI (blue). Scale bars: $100 \mu \mathrm{m}$. The percentage of (I) proliferative and (J) apoptotic trophoblasts in DCN-untreated/treated cultures, calculated as the number of $\mathrm{Ki67+}$ or cleaved cytokeratin- $18^{+}$cells into numbers of trophoblasts (K $7^{+}$cells). Results are presented as scatter plots with the indicated median values shown as horizontal lines. Statistical analyses between groups were performed using a nonparametric 2-tailed Mann-Whitney $t$ test.

remodeling of uterine arteries, but also control trophoblast survival and invasion (17). The effects of decorin production within the maternal-fetal interface is not well described; however, studies investigating its effects on trophoblastic in vitro models suggest that decorin negatively regulates cell proliferation and invasion (34). We therefore set out to examine the effects of uNK cell-derived decorin on trophoblast column growth using a first trimester placenta ex vivo culture system; cellular processes central to trophoblast column formation are critical for successful placental development and pregnancy. For these experiments, uNK cell conditioned media (CM) was harvested from uNK cells isolated from 3 obese women cultured over 18 hours; uNK cell purity was routinely greater than 90\% showing less than $3 \%$ CD $45^{-}$cell contamination (Figure 7A). Immunoblotting showed that uNK cells express a high molecular weight decorin in CM, indicative of varying levels of glycosylation (Figure 7B; full, uncut gels available in Supplemental Figure 4). Matrigel-imbedded placental explants cultured in control medium showed extensive trophoblast column outgrowth over 48 hours (Figure 7, C-E). In contrast, placental explants cultured in uNK cell CM exhibited impaired column outgrowth (Figure 7, D and E). Importantly, the inhibitory effect of uNK cell CM on column outgrowth was partially reversed by cotreatment with a function-neutralizing decorin antibody, suggesting that uNK cell-derived decorin impairs placental column formation (Figure 7, D and E).

To examine the direct effect of decorin on placental explant outgrowth, explants were cultured in the presence or absence of recombinant decorin. Ectopic decorin strongly inhibited trophoblast column outgrowth and cell invasion into surrounding matrix; in untreated control explants, trophoblast column growth and invasion were prominent (Figure 7, F and G). Since column outgrowth is regulated in part by trophoblast proliferation and survival, we examined the effect of decorin on these 2 cellular processes. Recombinant decorin did not alter trophoblast proliferation within intact cellular columns as measured by Ki67 positivity within keratin- $7^{+}$cells (Figure 7, $\mathrm{H}$ and I). However, decorin treatment led to profound increases in caspase-cleaved keratin-18 signal (a readout of cell apoptosis) in proliferating trophoblasts localized to cell columns and to senescent trophoblasts invading through Matrigel matrix (Figure 7, H and J). Taken together, these results suggest that decorin overexpression within the maternal-fetal interface affects placental development and health by inhibiting trophoblast column formation via mechanisms promoting cell apoptosis. Our data indicate that decorin overexpression by uNK cells in obesity may play a central role in impairing placental health.

\section{Discussion}

We have shown that maternal obesity instructs alterations in uNK cell composition and global gene expression within the uterine environment in early pregnancy. Notably, obesity leads to reductions in uNK cells within the decidual mucosa, and these cellular changes are tightly linked with impaired remodeling of uterine vasculature. Global gene expression strategies highlight the broad enrichment of ECM-signaling and growth factor-signaling pathways in uNK cells from obese pregnancies that mirror fibrotic and inflammatory signals associated with chronic tissue damage. Specifically, we provide evidence that hyperactive PDGFR signaling induces uNK cell survival signals and drives expression of COL3A1 and $D C N$. Importantly, our study identifies an obesity-driven mechanism imparted by uNK cell overexpression of decorin in controlling placental development and health. To our knowledge, this is the first study to establish mechanistic insight into the effects of obesity on uterine immune cell function and establishment of the maternal-fetal interface in humans. 
NK cells are large granular lymphocytes of the innate immune system and represent the first line of defense against pathogens (40). Unlike conventional NK cells found in the periphery, uNK cells are the dominant leukocyte population in the uterus in early pregnancy. In humans, uNK cell proportions greatly increase following ovulation and peak in number by 20 weeks of gestation, after which uNK cell numbers gradually regress until term (16). Evidence from studies in mice suggest that uNK cells represent a distinct tissue-specific lineage derived from tissue-resident NK cell precursors (41). However, the influx or tissuehoming potential of peripheral NK cells into the uterus may also significantly contribute to uNK cell composition during pregnancy; this possibility is supported by adoptive bone marrow transfer experiments in mice showing that bone marrow-to-uterus homing of NK cells is sufficient to rescue uterine arterial defects observed in NK cell-deficient mice (42). Our finding that CD56 $6^{\text {bright }} \mathrm{uNK}$ cell numbers are decreased in the uterus of obese women, in lieu of proliferative regulation, indicates that either a defect in NK cell homing to the uterus exists, or that disruptions in NK cell lineage commitment or survival are taking place. Our finding that uNK cell survival is impaired in obesity while proliferation remains unaltered suggests that obesity-derived factors establish a potentially hostile environment towards uNK cells, limiting their longevity. However, this interpretation requires some tempering, as Ki67 does not account for possible differences in cell cycle kinetics. Nonetheless, it will be important to phenotype peripheral blood NK cells as well as tissue residency markers of uNK cells (i.e., CD9) in addition to activating and inhibiting NK cell receptors (i.e., killer immunoglobulin-like receptors) to provide insight into obesity-directed functional changes in uNK cells. Future studies must also control for decidual sampling within the uterus (i.e., basalis versus parietalis), as possible spatial differences not controlled for in this study do require consideration. Interestingly, peripheral NK cell tissue-homing defects were observed in pregnant diabetic women, indicating that a similar effect may also be taking place in the condition of obesity (43).

Although unsupervised sample clustering using uNK cell global gene expression signatures did not distinguish between control or obese BMI groups, the use of high-CV gene signatures across our samples did instruct sample segregation into control and obese groups with relatively high accuracy (75\%). Incomplete sample segregation was somewhat expected as obesity itself may well be a heterogeneous condition composed of individuals having both healthy/unhealthy metabolism presenting without/with chronic inflammation (44). This latter point is crucial and is illustrated in our sample cohort in which obese women measuring healthy (low) serum CRP levels comprised $12 \%$ of women in our cohort. In contrast, CRP-elevated control/lean women made up just $7 \%$, indicating that although low-grade inflammation and obesity are not directly linked with each other, a strong correlation still exists (i.e., over $75 \%$ of obese women had elevated serum CRP). Moving forward, studies aimed at deciphering how obesity-related inflammation impacts immune cell function within the uterine environment in humans must consider adequate sample size number, and other variables likely contributing to heterogeneity such as ethnicity, lifestyle, age, and overall metabolic health.

Obesity-linked inflammation originating from adipose tissue is known to alter immune cell activity $(9,45)$. Therefore, we were surprised that minimal genes related to $\mathrm{NK} /$ immune cell function were differentially expressed in the G1/G2 obese group. Although immune cell-specific genes (i.e., CD38, LIME1, $N L R C 3, L R M P, I L 21 R$, and IL16) were shown to be underexpressed in the obese-enriched cohort, modest fold-change differences and pathway enrichment scores suggest that these alterations are not the major pathways instructing altered uNK cell biology. The finding that obesity led to enrichment of transcriptional programs related to growth factor binding and ECM organization was also unexpected. Notably, genes central to regulation of placental function and uterine arterial remodeling were highly enriched in uNK cells from obese subjects, and included multiple IGFBPs (IGFBP-3, -4, -5, -6, and -7) and TIMPs (TIMP-1, -2, and -3). IGFBP-mediated sequestration of IGF-I/II inhibits placental development by limiting promitogenic and proinvasion signaling in trophoblasts $(46,47)$. Likewise, TIMP-directed inhibition of trophoblast- and uNK cell-derived metalloproteinases obstructs trophoblast invasion and MMP9-directed vascular remodeling (48). In both systems, defects in either IGF or MMP function are clinically linked to pregnancy disorders $(49,50)$. Our observation that decidual artery remodeling was impaired in obese women is consistent with the implications of our transcriptional findings and establishes mechanistic insight as to why maternal obesity is associated with high-risk pregnancies.

Dynamic remodeling of the ECM is essential for development, wound healing, and tissue homeostasis, where chronic conditions arising from aberrant ECM deposition (i.e., fibrosis) lead to impairment in organ function. The increase in expression of genes associated with fibrotic processes in the obese G1/ 
G2 cluster is intriguing, as ECM composition influences immune cell behavior in inflamed tissues (51). For example, individual ECM proteins as well as complex three-dimensional ECM lattices convey specific information to immune cells to modulate cell infiltration into tissues, activation, and survival (36). Our finding that major gene pathways related to collagen production and organization are enriched in $\mathrm{uNK}$ cells from the obese G1/G2 group indicates that the uterine ECM is different in obese pregnancies compared with that in lean pregnancies. Specifically, the overexpression of multiple fibrillar collagens (COL1A1, COL1A2, COL3A1, COL4A5, COL5A1, COL5A2, COL6A1, COL6A2, and COL6A3) and collagen-modifying proteins $(D C N)$ suggests that uNK cells in obesity are directly contributing to an altered uterine microenvironment. The overexpression of PDGFRs that we have detected may be contributing to uNK cell-directed ECM production, but may also be playing a role in supporting uNK cell survival in a comparatively more hostile microenvironment. Localization of collagen-I protein to uNK cells adjacent to unremodeled uterine arteries supports previous work that highlights the role of dysregulated ECM production in disrupted tissue-remodeling processes in cancer and chronic diseases (52). Exactly how ECM alterations affect uNK cell or other uterine immune cell function remains to be examined.

uNK cells produce factors that regulate trophoblast biology $(53,54)$. Our finding that the $D C N$ gene is overexpressed in uNK cells from obese pregnancies identifies a potentially novel mechanism whereby maternal obesity negatively regulates placental development. Although uterine stromal-derived decorin has previously been shown to control trophoblast cell line invasion (34), our finding that decorin promotes apoptosis within distinct proliferative and invasive trophoblasts highlights a likely mechanism whereby decorin impedes placental development in vivo and establishes a potentially novel uNK cell-trophoblast interaction. Placental explants used in this study allow the interrogation of complex cellular processes (i.e., formation of proliferative trophoblast columns and differentiation of proliferative trophoblasts into invasive extravillous cells) that are not possible to interrogate using cell line models, and therefore provide a realistic model to study early placentation.

uNK cells are distinct immune cell subsets that play fundamental roles in orchestrating healthy pregnancy. Conditions or environments that instruct deviations from normal uNK cell biology may contribute to impaired angiogenesis and placental establishment implicated in causing pregnancy disorders. Our study demonstrates a potential mechanistic link driven by maternal obesity in inhibiting uNK cell-directed blood vessel remodeling and trophoblast health. Furthermore, this work establishes insight into the downstream effects of obesity-related chronic inflammation on uNK cell function in early pregnancy, thus highlighting a possible cellular effector contributing to obesity-driven poor pregnancy outcome. As obesity is an ever increasingly chronic condition in our population that poses serious consequences for reproductive health and fertility, understanding the cellular root causes affected by obesity is crucial. Investigation into how these obesity-related immunological changes within the uterus relate to pregnancy outcome will be of primary importance.

\section{Methods}

Patient recruitment and tissue collection. Blood, decidual, and placental tissues were obtained with approval from the Research Ethics Board on the use of human subjects, University of British Columbia (H1300640). All samples were collected from women (19 to 35 years of age) providing written informed consent undergoing elective terminations of pregnancy at British Columbia's Women's Hospital, Vancouver, Canada. First trimester decidual $(n=97)$ and placental tissues $(n=7)$, as well as whole-blood specimens (10 $\mathrm{ml}, n=90)$ were collected from participating women (gestational ages ranging from 5 to 13 weeks) having confirmed viable pregnancies by ultrasound-measured fetal heartbeat. Decidual tissue samples were selected based on the presence of a smooth uterine epithelial layer and a textured thick spongy underlayer. Serum was purified from whole blood using serum separator tubes following standard procedures. Patient clinical characteristics (i.e., height and weight) were additionally obtained to calculate BMI. All consenting women provided self-reported information via questionnaire regarding having diabetes, taking prescribed antiinflammatory or hypertensive medication, or having first-hand exposure to cigarette smoke. These selfreported data are shown in Supplemental Table 1.

ELISA measurements of serum CRP and leptin. Serum levels of CRP and leptin were determined using commercial ELISA kits following the manufacturers' protocols: Human High-Sensitivity (hs) CRP (Biovendor) and Quantikine Human Leptin (R\&D Systems). ELISA plates were read using a FLUOstar Optima plate reader (BMG LabTech). For hs-CRP and leptin measurements, serum was diluted 1:1,000 and 
1:100. Samples were excluded for further downstream analyses if serum CRP measurements exceeded 20 $\mu \mathrm{g} / \mathrm{ml}$, as this level may be indicative of an acute inflammatory response.

Decidual leukocyte isolation. Decidual tissues $(n=77)$ collected after elective termination of pregnancy were washed extensively in ice-cold PBS ( $\mathrm{pH} 7.4$ ), after which the tissues were finely minced using sterile razor blades and subjected to enzymatic digestion. Briefly, decidual leukocyte enrichment was performed by digesting minced decidual homogenates for 1 hour at $37^{\circ} \mathrm{C}$ in $4 \mathrm{ml}$ of 1:1 DMEM/F12 media (Gibco; 200 mM L-glutamine) containing $1 \times$ collagenase/hyaluronidase (10× stock; StemCell Technologies), 80 $\mu \mathrm{g} / \mathrm{ml}$ DNase I (Sigma-Aldrich), penicillin/streptomycin (Life Technologies), and 100-fold-diluted Antibiotic-Antimycotic Solution (Life Technologies). After digestion, the cell/tissue suspension was vortexed for 30 seconds and washed 2 times in HBSS/2\% FBS. Washed cells were sequentially pushed through $70-\mu \mathrm{m}$ and $40-\mu \mathrm{m}$ cell strainers to obtain single-cell suspensions, followed by discontinuous Percoll density gradient centrifugation (layered 40\%/80\%); enriched decidual leukocytes (routinely $>90 \% \mathrm{CD} 45^{+}$cells as assayed by flow cytometry) were collected from the middle cell layer located between the $40 \%$ and $80 \%$ Percoll. Decidual leukocytes were either (a) immediately used to isolate uterine NK cells for RNA extraction or short-term cell culture experiments, (b) stored in freezing media ( $90 \% \mathrm{FBS}+10 \% \mathrm{DMSO}$ ) to be used for downstream cell surface marker characterization via flow cytometry, or (c) subjected to cytokine expression, cytotoxicity, or migration experiments. Decidual NK cells were enriched via a combined negative selection and positive exclusion strategy using two customized immunolabeled magnetic bead cocktails following the manufacturer's protocol (formulated on the EasySep Human NK cell Enrichment Kit platform; StemCell Technology). The negative depletion antibody cocktail containing beads conjugated to antibodies directed against CD3, CD4, CD14, CD19, CD20, CD36, CD66b, CD123, and CD31 removed non-NK cell leukocytes and contaminating endothelial cells, leaving the remaining NK cell fraction "untouched." This NK-enriched fraction was next subjected to CD16 positive exclusion (EasySep Kit, StemCell Technologies) to remove $\mathrm{CD} 56^{+} \mathrm{CD} 16^{+} \mathrm{NK}$ cells. CD56 $6^{\text {bright }}$ cell purity was routinely greater than $95 \%$ as assayed by flow cytometry (Figure 2A). For experiments performed in Figures 6 and 7, uNK cell isolation was performed using only negative selection directly followed by a 2-hour preculture in complete RPMI media to remove contaminating stromal cells.

Flow cytometric analysis of extracellular markers. Following washing in FACS buffer ( $1 \times$ HBSS, 2\% FBS), $2 \times 10^{5}$ decidual leukocytes (per multicolor stain) were blocked with human FcR binding inhibitor (eBioscience) and incubated with the following extracellular markers: CD45-PerCP-eFluor 710 (1:100; clone HI30), CD56-FITC (1:25; clone MEM188), CD3-APC-eFluor 780 (1:100; clone SK7), CD16-APC (1:50; clone CB16), Fixable Viability Dye eFluor 780 (1:1,000; all from eBioscience) for 30 minutes at $4^{\circ} \mathrm{C}$. The cells were then fixed and permeabilized with fixation and permeabilization reagents from eBioscience, used according to the guidelines from the manufacturer. Afterwards, the cells were stained with Ki67-PECy7 (1:20; clone 20Raj1; eBioscience) for another 30 minutes at $4^{\circ} \mathrm{C}$. In a separate batch of experiments, decidual leukocytes were labeled with the following markers: CD45-PerCP-eFluor 710 (1:100), CD56-eFluor 450 (1:100; clone TULY56), CD3-APC-eFluor 780 (1:100), CD16-APC (1:50), Fixable Viability Dye eFluor 780 (1:1,000; all from eBioscience), PDGFR- $\beta$ /CD140b-PE (1:20; clone REA363; Miltenyi Biotec), and CD9-FITC (1:25; clone M-L13; BD Biosciences) for 30 minutes at $4^{\circ} \mathrm{C}$. Following staining, cells were resuspended in PBS and data were collected on the BD FACS Fortessa flow cytometer (BD Biosciences) and analyzed using FlowJo software (Tree Star Inc.).

Intracellular cytokine analysis, NK cell cytotoxicity assay, and PDGF-BB stimulation. Following washing in FACS buffer ( $1 \times$ HBSS, $2 \%$ FBS), $1 \times 10^{6}$ decidual leukocytes were resuspended in RPMI supplemented with $10 \% \mathrm{FBS}, 100 \mathrm{U} / \mathrm{ml}$ penicillin, and $100 \mathrm{mg} / \mathrm{ml}$ streptomycin, and cocultured with $\mathrm{K} 562$ cells at an effector:target ratio of $2: 1$ for 5 hours at $37^{\circ} \mathrm{C}$. Cells cultured in complete RPMI medium served as a negative control, whereas stimulation with $10 \mathrm{ng} / \mathrm{ml}$ phorbol 12-myristate 13 -acetate (PMA) and $500 \mathrm{ng} / \mathrm{ml}$ ionomycin served as a positive control. In a subset of experiments, decidual leukocytes were treated with $30 \mathrm{ng} / \mathrm{ml}$ recombinant human PDGF-BB (R\&D Systems); all cells were cultured in the presence of $3 \mu \mathrm{g} /$ $\mathrm{ml}$ brefeldin A and $2 \mu \mathrm{M}$ monensin (eBioscience), as well as with CD107a-PE (1:200; clone eBioH4A3; BD Biosciences). After incubation, decidual leukocytes were blocked with human FcR binding inhibitor (eBioscience) and labeled with antibodies directed against extracellular markers: CD45-PerCP-eFluor 710 (1:100; clone HI30), CD56-FITC (1:25; clone MEM188), CD3-APC-eFluor 780 (1:100), Fixable Viability Dye eFluor 780 (1:1,000; eBioscience), and CD107a-PE (1:20; BD Biosciences) for 30 minutes at $4^{\circ} \mathrm{C}$. Cells were then fixed and permeabilized with Fixation/Permeabilization Buffer (eBioscience). Afterwards, 
cells were stained with TNF- $\alpha$-APC (1:20; clone MAb11) and IFN- $\gamma$-AF700 (1:50; clone 4S.B3) for an additional 30 minutes at $4^{\circ} \mathrm{C}$ (both antibodies from eBioscience). Cells were resuspended in PBS and data were collected on the BD FACS Fortessa flow cytometer and analyzed using FlowJo software. For PDGFR pathway analysis, $1 \times 10^{6}$ negatively selected (StemSep; Stem Cell Technology) uNK cells were preplated for 2 hours in RPMI to remove contaminating fibroblasts, harvested, and starved in RPMI media for 5 hours at $37^{\circ} \mathrm{C}$ and $5 \% \mathrm{CO}_{2}$. Following starvation, uNK cells were stimulated with $100 \mathrm{ng} / \mathrm{ml} \mathrm{PDGF}-\mathrm{BB}$ for 10 minutes at $37^{\circ} \mathrm{C}$, placed on ice, centrifuged at $4^{\circ} \mathrm{C}$, and lysed in RIPA protein extraction buffer (20 $\mathrm{mM}$ Tris-HCl, $\mathrm{pH} 7.6,1 \%$ Triton X-100, 0.1\% SDS, 1\% NP-40, 1\% sodium deoxycholate, 5 mM EDTA, $50 \mathrm{mM} \mathrm{NaCl}$ ). For examining the effects of PDGF-BB on gene expression, $1 \times 10^{6} \mathrm{uNK}$ cells were treated with $30 \mathrm{ng} / \mathrm{ml}$ PDGF-BB in RPMI medium containing 1\% FBS, $5 \mathrm{ng} / \mathrm{ml}$ IL-15 (BioLegend), $55 \mathrm{nM}$ 2-ME, $1 \mathrm{mM}$ sodium pyruvate (Life Technologies), penicillin/streptomycin (Life Technologies), and AntibioticAntimycotic Solution (Life Technologies) for 12 hours at $37^{\circ} \mathrm{C}$ and $5 \% \mathrm{CO}_{2}$.

Decidual NK cell migration assay. Decidual NK cell migration was assayed using 5.0- $\mu$ m-pore Boyden chamber Transwell inserts fitted into in 24 -well plates (Corning). Decidual leukocytes $\left(2 \times 10^{5}\right.$ cells) in 100 $\mu 1$ of serum-free media were added to the top insert chamber; inserts were placed into 24-well plates containing $600 \mu \mathrm{L}$ of RPMI 1640 media either (a) supplemented with 10\% FBS, (b) supplemented with $30 \mathrm{ng} /$ $\mathrm{ml}$ of PDGF-BB, or (c) serum-free media alone. Assays were performed at $37^{\circ} \mathrm{C}$ and $5 \% \mathrm{CO}_{2}$ for 3 hours. Afterwards, cells that passed through the filter were collected and stained with the indicated antibodies: CD45 (PerCP-eFluor 710; clone HI30), CD3 (PE-Cy7; clone UCHT1), CD56 (FITC; clone MEM188), CD16 (APC; clone CB16), and Fixable Viability Dye eFluor 780 (eBioscience). Absolute cell counting was determined with an internal control (123count eBeads; eBioscience) following the manufacturer's protocol using the BD Fortessa flow cytometer.

RNA purification and microarray expression analysis. Total RNA was prepared from immunomagnetic bead-enriched uNK cells using the RNAqueous Total RNA Isolation Kit following the manufacturer's instructions (Ambion). RNA purity was confirmed using a NanoDrop Spectrophotometer (Thermo Fisher Scientific) and by running RNA samples on an Agilent 2100 Bioanalyzer (Agilent Technologies). For microarray analysis, only RNA samples having an RNA integrity number (RIN) greater than 7.0 were used. For microarray gene expression analysis, 24 biotin-labeled cRNA samples were generated using TargetAmp labeling kits (13 control and 11 obese uNK cell samples) and were hybridized onto HumanHT-12 v4 Expression BeadChips (Illumina). The HumanHT-12 v4 Expression BeadChips target greater than 47,000 probes derived from the NCBI RefSeq Release 38 (November 7, 2009).

Data preprocessing and identification of signature genes and pathways. Preprocessing and statistical analyses of raw Illumina IDAT files were performed within the R statistical environment (v2.15.2) using the limma library beadarray $(\mathrm{v} 3.14 .3)(55,56)$ packages of the Bioconductor open-source project $(57)$. Raw expression data were log transformed and quantile normalized using the normaliselllumina() function. The probewise quality grade was estimated by the illuminaHumanv4PROBEQUALITY() function for 48,107 probes in the raw microarray data, resulting in 34,476 high-quality probes (quality grade "Good" or "Perfect") for the following analysis, after removing 13,631 poor-quality probes (grade "Bad") and probes that could not be mapped to any genes (labeled as "No match"). Batch effect was corrected using the surrogate variable analysis SVA () function. PCA and surrogate variable analysis were performed by the built-in svd() function and the sva package in R. After preprocessing, mRNA expression was analyzed using the univariate linear model. All model-based $t$ tests were corrected using empirical Bayes moderation of the SE, followed by FDR adjustment for multiple testing (58). Genes that showed significant differential expression were filtered based on a $q$ value less than 0.001 . Enrichment of pathways in high-CV genes or genes that were shown to be differentially expressed between $\mathrm{G} 1 / 2$ and G3/4 clusters were identified and annotated using ToppGene software (31). Processed data were plotted using gplots and ape bioconductor packages in $\mathrm{R}$.

$q P C R$ analysis. One microgram of RNA was reverse-transcribed using a first-strand cDNA synthesis kit (QuantaBiosciences Inc.) and analyzed by qPCR $(\triangle \Delta C T)$, using Perfecta SYBR Green FastMix (QuantaBiosciences) on an ABI 7500 Sequence Detection System (Life Technologies). Forward and reverse primer sets were as follows: PDGFRA (F: 5'-TTCCTCTGCCTGACATTGAC-3', R: 5'-GTCTTCAATGGTCTCGTCCTC-3'); PDGFRB (F: 5'-CCACACTCCTTGCCCTTTAAG-3', R: 5'-CTCACAGACTCAATCACCTTCC-3'); COL1A1 (F: 5'-TACAGCGTCACTGTCGATGG-3', R: 5'-TCAATCACTGTCTTGCCCCAG-3'); COL3A1 (F: 5'-AAGTCAAGGAGAAAGTGGTCG-3', R: 5'-CTCGTTCTCCATTCTTACCAGG-3'); COL6A3 (F： 5'-GAGATGTTGGGATTCGAGGG-3', 
R: 5'-GTTTCTCCTTTGGGTCCTCTC-3'); DCN (F: 5'-TGATGCAGCTAGCCTGAAAGG-3', R: 5'-AGGCGTGTTGGCCAGAGAG-3'); GAPDH (F: 5'-AGGGCTGCTTTTAACTCTGGT -3', R: 5'-CCCCACTTGATTTTGGAGGGA -3'). All raw data were analyzed using Sequence Detection System software version 2.1 (Life Technologies). The threshold cycle (CT) values were used to calculate relative RNA expression levels. Values were normalized to endogenous GAPDH transcripts.

Immunohistochemistry and IF microscopy. Uterine decidual specimens (11-13 weeks of gestation; $n=20$ ) and Matrigel-imbedded placental explants (5-8 weeks of gestation, $n=28$ ) were fixed in $2 \%$ paraformaldehyde overnight at $4{ }^{\circ} \mathrm{C}$. Tissues and explants were paraffin embedded and serially sectioned at $5 \mu \mathrm{m}$ onto glass slides. IF was performed as described previously (59). Briefly, tissues underwent antigen retrieval by heating slides in a microwave for five 2-minute intervals in citrate buffer $(\mathrm{pH}$ 6.0). Sections were incubated with sodium borohydride for 5 minutes at room temperature, followed by $0.1 \%$ Triton X-100 permeabilization for 5 minutes at room temperature. Slides were blocked in $5 \%$ normal goat serum $/ 0.1 \%$ saponin for 1 hour at room temperature, and incubated with combinations of the indicated antibodies overnight at $4^{\circ} \mathrm{C}$ : mouse monoclonal CD56 (1:200; clone 123C3; Cell Signaling Technology); rabbit monoclonal CD56 (1:100; clone EP2567Y; Abcam); mouse monoclonal SMA (1:400; clone 1A4; Dako); mouse monoclonal CD31 (1:400; clone JC70A; Dako); rabbit polyclonal PDGFR- $\alpha$ (1:200; Upstate); rabbit monoclonal keratin-7 (1:75; clone SP52; Ventana); mouse monoclonal keratin-7 (1:200; clone C46; Santa Cruz Biotechnology); rabbit monoclonal Ki67 (1:100; clone SP6; Thermo Fisher Scientific); mouse monoclonal caspase-cleaved keratin-18 (1:25; clone M30; Roche); rabbit monoclonal cleaved caspase-3 (1:200; clone 5A1E; Cell Signaling Technology); and mouse monoclonal decorin (1:100; Ab54728; Abcam). Slides were incubated with Alexa Fluor 568/488-conjugated goat anti-rabbit and goat anti-mouse secondary antibodies (Life Technologies) for 1 hour at room temperature. Glass coverslips were mounted onto slides using DAPI mounting media (Life Technologies) and slides were imaged using a 20× Plan-Apochromat $/ 0.80$ NA or 40× Plan-Apochromat oil/1.4 NA objective (Carl Zeiss). An ApoTome.2 structured illumination device (Carl Zeiss) set at full Z-stack mode and 5 phase images was used in imaging Figures 2I and 6C. For quantification of uNK cell numbers, artery smooth muscle thickness, vessel:lumen ratio, arteries per field of view, proportion of cleaved caspase- $3 \mathrm{CD} 56^{+}$cells and mean decorin channel intensity, 10 images per tissue section (obtained using a $20 \times$ objective) were used to calculate values. uNK cell quantities were calculated by $\mathrm{CD} 56^{+} / \mathrm{DAPI}^{+}$cells per field of view averaged over 10 images. CD56 and cleaved caspase- 3 signal hierarchical thresholding, based on DAPI ${ }^{+}$cells meeting CD56 and cleaved caspase- 3 fluorescence intensity thresholds, was used to calculate $\mathrm{CD} 56^{+}$and $\mathrm{CD} 56^{+} /$cleaved caspase- $3^{+}$proportions. Smooth muscle (SM) artery thickness was calculated by averaging 8 linear measurements $(\mu \mathrm{m})$ of SM artery signal that encapsulated an artery (from outermost SM artery signal to SM artery signal proximal to inner arterial lumen); vessel:lumen ratios were calculated by dividing the SM artery area $\left(\mu \mathrm{m}^{2}\right)$ surrounding an artery into the artery lumen area $\left(\mu \mathrm{m}^{2}\right)$. Mean decorin signal was measured using raw EGFP channel fluorescence intensity readings using a circular drawing tool to isolate and quantify decorin signal in and within $5 \mu \mathrm{m}$ of uNK cells (defined by CD56 signal); 200 uNK cells per sample were quantified. Images were obtained using an Axiocam 506 monochrome digital camera and processed and analyzed using ZenPro software (Carl Zeiss).

Cell lysis and immunoblot analysis. Cells were washed in ice-cold PBS and incubated in RIPA cell extraction buffer supplemented with $10 \mathrm{mM} \mathrm{Na}_{3} \mathrm{VO}_{4}, 10 \mathrm{mM} \mathrm{NaF}, 2 \mathrm{mM}$ PMSF, and an appropriate dilution of Complete Mini, EDTA-free protease inhibition cocktail tablets (Roche). Protein concentrations were determined using a BCA kit (Thermo Fisher Scientific). For immunoblotting, $30 \mu \mathrm{g}$ of cell protein lysate or conditioned media was resolved by SDS-PAGE and transferred to nitrocellulose membranes. The membranes were probed using monoclonal antibodies directed against phosphorylated p44/42 MAPK (p-Erk1/2, Thr202/Tyr204), p44/42 MAPK (Erk1/2), phosphorylated Akt/PKB (p-Akt, Ser473), Akt, phosphorylated PDGFR- $\alpha / \beta$ (p-PDGFR- $\alpha / \beta$, Tyr 849/857) (all from Cell Signaling Technology), PDGFR- $\alpha$ (clone C-20; Santa Cruz Biotechnology), and a goat polyclonal antibody targeting decorin (AF143; R\&D Systems). The blots were stripped and reprobed with an HRP-conjugated monoclonal antibody directed against mouse $\beta$-actin (Santa Cruz Biotechnology).

Placental villous explant assay. Ex vivo placental villous cultures were established as previously described (59). Briefly, placental villi from placentae $(n=7)$ at 5 to 8 weeks of gestation obtained from patients undergoing elective termination of pregnancy were dissected, washed in cold PBS, and imbedded in Millicell cell culture inserts (0.4- $\mu \mathrm{m}$ pores, $12-\mathrm{mm}$ diameter; EMD Millipore) containing $200 \mu \mathrm{l}$ of Growth Factor-Reduced, Phenol Red-Free Matrigel (BD Biosciences). Explants, containing $400 \mu 1$ DMEM/ 
F12 1:1 (200 mM L-glutamine) in the outer chamber, were allowed to establish overnight in a humidified $37^{\circ} \mathrm{C}$ trigas incubator at $3 \%$ oxygen, $5 \% \mathrm{CO}_{2}$. Concurrent with explant establishment, negatively selected uNK cells from the same tissue sample were cultured for 18 hours in RMPI containing $1 \% \mathrm{FBS}, 5 \mathrm{ng} / \mathrm{ml}$ IL-15 (BioLegend), $55 \mathrm{nM}$ 2-ME, $1 \mathrm{mM}$ sodium pyruvate (Life Technologies), penicillin/streptomycin (Life Technologies) and Antibiotic-Antimycotic (diluted 100-fold). Following culture, uNK cell CM (and complete RPMI media as negative control) was collected, centrifuged, and concentrated 10 -fold in $10-\mathrm{kDa}$ concentrating filters (Millipore). Following 24 hours of culture, explants were cultured in $200 \mu 1 \mathrm{DMEM} /$ F12 1:1 media containing: (a) uNK cell CM (diluted 1/10); (b) RPMI media containing 1\% FBS, 5 ng/ $\mathrm{ml}$ IL-15, and $55 \mathrm{nM} 2-\mathrm{ME}$; (c) $20 \mu \mathrm{g} / \mathrm{ml}$ recombinant human decorin (ab169898; Abcam); or (d) media alone for an additional 48 hours. Endogenous decorin in uNK CM was functionally blocked using $10 \mu \mathrm{g} /$ $\mathrm{ml}$ anti-decorin neutralizing antibody (AF143; R\&D Systems); uNK CM was pretreated for 1 hour at $37^{\circ} \mathrm{C}$ with anti-decorin antibody prior to explant culture. All explant media were supplemented with penicillin/ streptomycin and Antibiotic-Antimycotic Solution. Growing explants were imaged at indicated times using a Nikon SMZ 7454T triocular dissecting microscope outfitted with a digital camera. Extravillous trophoblast (EVT) outgrowths were measured by ImageJ software (NIH). Length of outgrowths was determined by dividing the mean column length ( 5 measurements per explant) at 48 hours into the mean column length at 0 hours. Each treatment was conducted in duplicate and each experiment was performed 3 or 4 times using 7 different placentae.

Statistics. Flow cytometry, microscopy image quantification, and cell migration data are reported as median values and IQRs. qPCR gene expression data are presented as mean values \pm standard deviation. All calculations were carried out using GraphPad Prism software. For single comparisons, either MannWhitney nonparametric unpaired 2-tailed $t$ tests or 2-tailed Wilcoxon signed-rank $t$ tests (Figure 6) were performed. The differences were accepted as significant at $P$ less than 0.05 . For microarray gene expression statistical analyses, please refer to the Data Preprocessing section in methods.

Study approval. The present study in humans was reviewed and approved by the Research Ethics Board on the use of human subjects, University of British Columbia, Vancouver, British Columbia, Canada (human ethics certificate number H13-00640).

Accession number. The GEO accession number for the data reported in this paper is GSE75091.

\section{Author contributions}

AGB designed the research. SP, BC, YM, KC, LD, and AGB performed experiments and analyzed data. SP and AGB wrote the paper. All authors read and approved the manuscript.

\section{Acknowledgments}

The authors extend their sincere gratitude for the hard work of the staff at British Columbia's Women's Hospital's CARE Program for recruiting participants to our study, and thank Megan K. Barker, Megan Levings, and Laura Sly for their critical readings of the manuscript. We are thankful to John Priatel for his generous gift of the K562 target cell line and helpful discussions. We are additionally grateful to Justin Li (Accura Sciences) for his assistance in analyzing our microarray gene expression data sets. This work was supported by a SickKids Foundation New Investigator Grant (to AGB) and a Canadian Institutes of Health Research Open Operating Grant number 201403MOP-325905-CIA-CAAA (to AGB).

Address correspondence to: Alexander G. Beristain, Child and Family Research Institute, Department of Obstetrics and Gynecology, The University of British Columbia, Vancouver, British Columbia, Canada V5Z 4H4. Phone: 604.875.3573; E-mail: aberista@mail.ubc.ca.

1. Vahratian A. Prevalence of overweight and obesity among women of childbearing age: results from the 2002 National Survey of Family Growth. Matern Child Health J. 2009;13(2):268-273.

2. McGuire S. Shields M., Carroll M.D., Ogden C.L. Adult obesity prevalence in Canada and the United States. NCHS data brief no. 56, Hyattsville, MD: National Center for Health Statistics, 2011. Adv Nutr. 2011;2(4):368-369.

3. Goldenberg RL, Culhane JF, Iams JD, Romero R. Epidemiology and causes of preterm birth. Lancet. 2008;371(9606):75-84.

4. Kim YM, et al. Failure of physiologic transformation of the spiral arteries in the placental bed in preterm premature rupture of membranes. Am J Obstet Gynecol. 2002;187(5):1137-1142.

5. Avagliano L, Marconi AM, Romagnoli S, Bulfamante GP. Abnormal spiral arteries modification in stillbirths: the role of maternal prepregnancy body mass index. J Matern Fetal Neonatal Med. 2012;25(12):2789-2792. 
6. Hayes EK, et al. Trophoblast invasion and blood vessel remodeling are altered in a rat model of lifelong maternal obesity. Reprod Sci. 2014;21(5):648-657.

7. Parker VJ, Solano ME, Arck PC, Douglas AJ. Diet-induced obesity may affect the uterine immune environment in early-mid pregnancy, reducing NK-cell activity and potentially compromising uterine vascularization. Int J Obes (Lond). 2014;38(6):766774.

8. Gregor MF, Hotamisligil GS. Inflammatory mechanisms in obesity. Annu Rev Immunol. 2011;29:415-445.

9. Wensveen FM, et al. NK cells link obesity-induced adipose stress to inflammation and insulin resistance. Nat Immunol. 2015;16(4):376-385.

10. Yang $\mathrm{H}$, et al. Obesity increases the production of proinflammatory mediators from adipose tissue $\mathrm{T}$ cells and compromises TCR repertoire diversity: implications for systemic inflammation and insulin resistance. J Immunol. 2010;185(3):1836-1845.

11. Wu L, et al. Activation of invariant natural killer T cells by lipid excess promotes tissue inflammation, insulin resistance, and hepatic steatosis in obese mice. Proc Natl Acad Sci USA. 2012;109(19):E1143-E1152.

12. $\mathrm{Wu} \mathrm{D}$, et al. Eosinophils sustain adipose alternatively activated macrophages associated with glucose homeostasis. Science. 2011;332(6026):243-247.

13. Lynch L et al. Adipose tissue invariant NKT cells protect against diet-induced obesity and metabolic disorder through regulatory cytokine production. Immunity. 2012;37(3):574-587.

14. Molofsky AB, et al. Innate lymphoid type 2 cells sustain visceral adipose tissue eosinophils and alternatively activated macrophages. JExp Med. 2013;210(3):535-549.

15. Lee J. Adipose tissue macrophages in the development of obesity-induced inflammation, insulin resistance and type 2 diabetes. Arch Pharm Res. 2013;36(2):208-222.

16. Lima PD, Zhang J, Dunk C, Lye SJ, Croy BA. Leukocyte driven-decidual angiogenesis in early pregnancy. Cell Mol Immunol. 2014;11(6):522-537.

17. Wallace AE, Fraser R, Cartwright JE. Extravillous trophoblast and decidual natural killer cells: a remodelling partnership. Hum Reprod Update. 2012;18(4):458-471.

18. Le Bouteiller P. Human decidual NK cells: unique and tightly regulated effector functions in healthy and pathogen-infected pregnancies. Front Immunol. 2013;4:404.

19. Felker AM, Chen Z, Foster WG, Croy BA. Receptors for non-MHC ligands contribute to uterine natural killer cell activation during pregnancy in mice. Placenta. 2013;34(9):757-764.

20. Hofmann AP, Gerber SA, Croy BA. Uterine natural killer cells pace early development of mouse decidua basalis. Mol Hum Reprod. 2014;20(1):66-76.

21. Kim M, et al. VEGF-A regulated by progesterone governs uterine angiogenesis and vascular remodelling during pregnancy. EMBO Mol Med. 2013;5(9):1415-1430.

22. Xiong S, et al. Maternal uterine NK cell-activating receptor KIR2DS1 enhances placentation. J Clin Invest. 2013;123(10):42644272 .

23. Kieckbusch J, Gaynor LM, Moffett A, Colucci F. MHC-dependent inhibition of uterine NK cells impedes fetal growth and decidual vascular remodelling. Nat Commun. 2014;5:3359.

24. Lash GE, et al. Expression of angiogenic growth factors by uterine natural killer cells during early pregnancy. J Leukoc Biol. 2006;80(3):572-580.

25. Ball E, Bulmer JN, Ayis S, Lyall F, Robson SC. Late sporadic miscarriage is associated with abnormalities in spiral artery transformation and trophoblast invasion. J Pathol. 2006;208(4):535-542.

26. Hiby SE, et al. Combinations of maternal KIR and fetal HLA-C genes influence the risk of preeclampsia and reproductive success. J Exp Med. 2004;200(8):957-965.

27. Pijnenborg R, Vercruysse L, Hanssens M. The uterine spiral arteries in human pregnancy: facts and controversies. Placenta. 2006;27(9-10):939-958.

28. Koenig W. High-sensitivity C-reactive protein and atherosclerotic disease: from improved risk prediction to risk-guided therapy Int J Cardiol. 2013;168(6):5126-5134.

29. Ridker PM. Cardiology Patient Page. C-reactive protein: a simple test to help predict risk of heart attack and stroke. Circulation. 2003;108(12):e81-e85.

30. Chen J, Aronow BJ, Jegga AG. Disease candidate gene identification and prioritization using protein interaction networks. $B M C$ Bioinformatics. 2009;10:73.

31. Chen J, Bardes EE, Aronow BJ, Jegga AG. ToppGene Suite for gene list enrichment analysis and candidate gene prioritization. Nucleic Acids Res. 2009;37(Web Server issue):W305-W311.

32. King JC. Maternal obesity, metabolism, and pregnancy outcomes. Annu Rev Nutr. 2006;26:271-291.

33. Chen S, Young MF, Chakravarti S, Birk DE. Interclass small leucine-rich repeat proteoglycan interactions regulate collagen fibrillogenesis and corneal stromal assembly. Matrix Biol. 2014;35:103-111.

34. Iacob D, et al. Decorin-mediated inhibition of proliferation and migration of the human trophoblast via different tyrosine kinase receptors. Endocrinology. 2008;149(12):6187-6197.

35. Khan GA, Girish GV, Lala N, Di Guglielmo GM, Lala PK. Decorin is a novel VEGFR-2-binding antagonist for the human extravillous trophoblast. Mol Endocrinol. 2011;25(8):1431-1443.

36. Duarte S, Baber J, Fujii T, Coito AJ. Matrix metalloproteinases in liver injury, repair and fibrosis. Matrix Biol. 2015;44-46:147-156.

37. Malavaki CJ, et al. Imatinib as a key inhibitor of the platelet-derived growth factor receptor mediated expression of cell surface heparan sulfate proteoglycans and functional properties of breast cancer cells. FEBS J. 2013;280(10):2477-2489.

38. Malabanan KP, Sheahan AV, Khachigian LM. Platelet-derived growth factor-BB mediates cell migration through induction of activating transcription factor 4 and tenascin-C. Am J Pathol. 2012;180(6):2590-2597.

39. Hayes BJ, et al. Activation of platelet-derived growth factor receptor alpha contributes to liver fibrosis. PLoS ONE. 2014;9(3):e92925.

40. Rätsep MT, Felker AM, Kay VR, Tolusso L, Hofmann AP, Croy BA. Uterine natural killer cells: supervisors of vasculature construction in early decidua basalis. Reproduction. 2015;149(2):R91-102. 
41. Sojka DK, et al. Tissue-resident natural killer (NK) cells are cell lineages distinct from thymic and conventional splenic NK cells. Elife. 2014;3:e01659.

42. Zhang JH, Yamada AT, Croy BA. DBA-lectin reactivity defines natural killer cells that have homed to mouse decidua. Placenta. 2009;30(11):968-973.

43. Seaward AV, Burke SD, Ramshaw H, Smith GN, Croy BA. Circulating CD $56^{+}$cells of diabetic women show deviated homing potential for specific tissues during and following pregnancy. Hum Reprod. 2011;26(7):1675-1684

44. Marques-Vidal P, Velho S, Waterworth D, Waeber G, von Känel R, Vollenweider P. The association between inflammatory biomarkers and metabolically healthy obesity depends of the definition used. Eur J Clin Nutr. 2012;66(4):426-435.

45. Magalhaes I, et al. Mucosal-associated invariant T cell alterations in obese and type 2 diabetic patients. J Clin Invest. 2015;125(4):1752-1762.

46. Knöfler M. Critical growth factors and signalling pathways controlling human trophoblast invasion. Int J Dev Biol. 2010;54(23):269-280.

47. Sferruzzi-Perri AN, et al. An obesogenic diet during mouse pregnancy modifies maternal nutrient partitioning and the fetal growth trajectory. FASEB J. 2013;27(10):3928-3937.

48. Plaks V, et al. Matrix metalloproteinase-9 deficiency phenocopies features of preeclampsia and intrauterine growth restriction. Proc Natl Acad Sci USA. 2013;110(27):11109-11114.

49. Palei AC, et al. Association between matrix metalloproteinase (MMP)-2 polymorphisms and MMP-2 levels in hypertensive disorders of pregnancy. Exp Mol Pathol. 2012;92(2):217-221.

50. Forbes K, Westwood M, Baker PN, Aplin JD. Insulin-like growth factor I and II regulate the life cycle of trophoblast in the developing human placenta. Am J Physiol Cell Physiol. 2008;294(6):C1313-C1322.

51. Zimmermann HW, Tacke F. Modification of chemokine pathways and immune cell infiltration as a novel therapeutic approach in liver inflammation and fibrosis. Inflamm Allergy Drug Targets. 2011;10(6):509-536.

52. Campbell NE, Kellenberger L, Greenaway J, Moorehead RA, Linnerth-Petrik NM, Petrik J. Extracellular matrix proteins and tumor angiogenesis. J Oncol. 2010;2010:586905.

53. Robson A, et al. Uterine natural killer cells initiate spiral artery remodeling in human pregnancy. FASEB J. 2012;26(12):48764885.

54. Monk JM, Leonard S, McBey BA, Croy BA. Induction of murine spiral artery modification by recombinant human interferongamma. Placenta. 2005;26(10):835-838.

55. Smyth GK. Linear models and empirical bayes methods for assessing differential expression in microarray experiments. Stat Appl Genet Mol Biol. 2004;3:Article3.

56. Smyth GK, Speed T. Normalization of cDNA microarray data. Methods. 2003;31(4):265-273.

57. Gentleman RC, et al. Bioconductor: open software development for computational biology and bioinformatics. Genome Biol. 2004;5(10):R80

58. Storey JD, Tibshirani R. Statistical methods for identifying differentially expressed genes in DNA microarrays. Methods Mol Biol. 2003;224:149-157.

59. Aghababaei M, Perdu S, Irvine K, Beristain AG. A disintegrin and metalloproteinase 12 (ADAM12) localizes to invasive trophoblast, promotes cell invasion and directs column outgrowth in early placental development. Mol Hum Reprod. $2014 ; 20(3): 235-249$ 